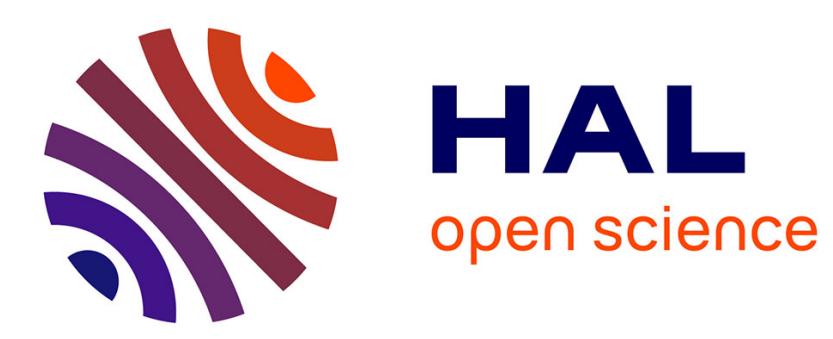

\title{
An invariant vortex-force theory related to classical far-field analyses in transonic flows
}

Camille Fournis, Didier Bailly, Renato Tognaccini

\section{To cite this version:}

Camille Fournis, Didier Bailly, Renato Tognaccini. An invariant vortex-force theory related to classical far-field analyses in transonic flows. AIAA AVIATION 2021, Jun 2021, WASHINGTON, United States. hal-03396061

\section{HAL Id: hal-03396061 https://hal.science/hal-03396061}

Submitted on 22 Oct 2021

HAL is a multi-disciplinary open access archive for the deposit and dissemination of scientific research documents, whether they are published or not. The documents may come from teaching and research institutions in France or abroad, or from public or private research centers.
L'archive ouverte pluridisciplinaire HAL, est destinée au dépôt et à la diffusion de documents scientifiques de niveau recherche, publiés ou non, émanant des établissements d'enseignement et de recherche français ou étrangers, des laboratoires publics ou privés. 


\title{
An invariant vortex-force theory related to classical far-field analyses in transonic flows
}

\author{
Camille Fournis* and Didier Bailly ${ }^{\dagger}$ \\ ONERA-The French Aerospace Lab, 92190 Meudon, France \\ Renato Tognaccini \\ Università di Napoli Federico II, Napoli, 80125, Italia
}

\begin{abstract}
Recent studies about the Lamb vector have led to the development of the vortex-force theory, a formulation able to predict the aerodynamic force in compressible flows and decompose it into lift, lift-induced drag and profile drag. Here, a revised formulation of the vortex-force theory developed at ONERA in collaboration with the University of Naples is presented and applied to steady transonic flows. In the mathematical developments, special care is given to the presence of shock wave discontinuities within the flow field. The equivalence between the new definition, the Kutta-Joukowski lift theorem, Maskell's lift-induced drag formula and Betz's profile drag formula is emphasized. The revised formulation also presents several practical advantages: the decomposition is naturally independent of the reference point chosen for the calculation of moment transformations, and the computation can be performed in a better refined part of the grid. The formulation is finally tested on the NASA Common Research Model (CRM) wing-fuselage configuration in cruise flight conditions.
\end{abstract}

\section{Nomenclature}

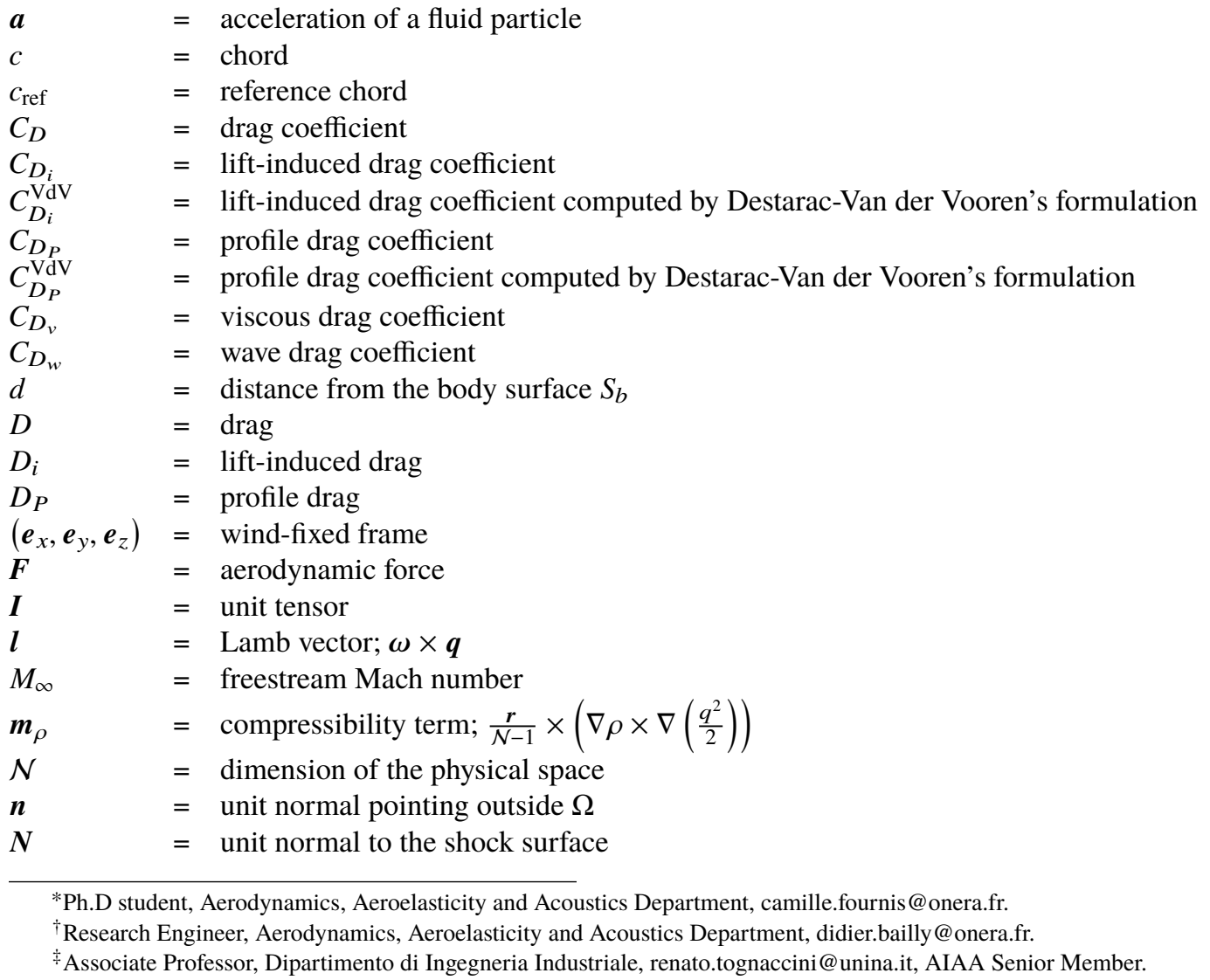




\begin{tabular}{|c|c|c|}
\hline $\boldsymbol{n}_{\mathrm{sw}}$ & $=$ & unit normal pointing outside $\Omega_{\mathrm{sw}}$ \\
\hline$p$ & $=$ & pressure \\
\hline$p_{t}$ & $=$ & total pressure; $p+\frac{1}{2} \rho q^{2}$ \\
\hline$p_{t \infty}$ & $=$ & freestream total pressure; $p_{\infty}+\frac{1}{2} \rho_{\infty} U_{\infty}^{2}$ \\
\hline$q$ & $=$ & velocity vector; $\left(U_{\infty}+u\right) \boldsymbol{e}_{x}+v \boldsymbol{e}_{y}+w \boldsymbol{e}_{z}$ \\
\hline$q^{2}$ & $=$ & velocity squared; $\left(U_{\infty}+u\right)^{2}+v^{2}+w^{2}$ \\
\hline $\boldsymbol{r}$ & $=$ & position vector \\
\hline $\operatorname{Re}$ & $=$ & Reynolds number \\
\hline$s$ & $=$ & entropy \\
\hline$S_{e}$ & $=$ & external boundary \\
\hline$S_{b}$ & $=$ & body surface \\
\hline SW & $=$ & shock wave \\
\hline $\boldsymbol{U}_{\infty}$ & $=$ & freestream velocity; $U_{\infty} \boldsymbol{e}_{x}$ \\
\hline$W$ & $=$ & wake plane \\
\hline$(x, y, z)$ & $=$ & wind-fixed coordinate system \\
\hline$\alpha$ & $=$ & angle of attack \\
\hline$\gamma$ & $=$ & ratio of specific heats \\
\hline$\delta \boldsymbol{q}$ & $=$ & perturbation velocity vector; $u \boldsymbol{e}_{x}+v \boldsymbol{e}_{y}+w \boldsymbol{e}_{z}$ \\
\hline$\Omega$ & $=$ & fluid domain \\
\hline$\omega$ & $=$ & vorticity vector; $\nabla \times \boldsymbol{q}$ \\
\hline$\Omega_{v}$ & $=$ & boundary layer region \\
\hline$\Omega_{\mathrm{sW}}$ & $=$ & shock wave region \\
\hline$\rho$ & $=$ & density \\
\hline$\rho_{\infty}$ & $=$ & freestream density \\
\hline$\tau$ & $=$ & viscous-stress tensor \\
\hline$\nabla$ & $=$ & gradient operator; $(\partial / \partial x) \boldsymbol{e}_{x}+(\partial / \partial y) \boldsymbol{e}_{y}+(\partial / \partial z) \boldsymbol{e}_{z}$ \\
\hline
\end{tabular}

\section{Introduction}

INCE the birth of aviation in the late nineteenth and early twentieth century, the need for ever lighter and less fuel consuming aircraft has kept research going on the topic of accurate aerodynamic force prediction. Indeed, the aerodynamic drag acting on an aircraft has a significant influence on its global performance and fuel consumption. This justifies the need for formulations that can provide faithful force predictions and a physical understanding of the phenomena occuring in the vicinity of the aircraft. Indeed, such tools may further enable aeronautical engineers to improve their design.

The aerodynamic force can be calculated by the near-field and the far-field approaches. The former consists in integrating the pressure and shear stresses acting on the body skin $S_{b}$ whereas the latter is based on the momentum balance in a control volume of fluid $\Omega$ and its external boundary $S_{e}$ (see Fig 1 ):

$$
\boldsymbol{F}=-\oint_{S_{b}}(-p \boldsymbol{I}+\boldsymbol{\tau}) \cdot \boldsymbol{n} \mathrm{d} S=-\int_{\Omega} \rho \boldsymbol{a} \mathrm{d} v+\oint_{S_{e}}(-p \boldsymbol{I}+\boldsymbol{\tau}) \cdot \boldsymbol{n} \mathrm{d} S
$$

where $\boldsymbol{a}$ is the acceleration of a fluid particle, $\boldsymbol{\tau}$ is the viscous-stress tensor and $\boldsymbol{n}$ is the unit normal to the surface pointing outside the control volume.

The near-field approach only provides a mechanical breakdown of the drag into friction and pressure drag. This decomposition is in fact not suited to develop a physical understanding of the origin of the drag and cannot be used to improve the design of airplanes. Moreover, the discrepancies generated by the grid close to the body skin in numerical computations may undermine the reliability of the total drag prediction. On the contrary, far-field methods provide a phenomenological decomposition of the aerodynamic force related to various flow structures: trailing vortices, jets, viscous wakes and shock waves. Hence, for non-propelled aircraft, the total drag is the sum of three contributions; the lift-induced drag, the viscous drag and the wave drag: $C_{D}=C_{D_{i}}+C_{D_{v}}+C_{D_{w}}$. Furthermore, it is possible to estimate the spurious drag present in numerical simulations.

The first far-field approach was developed independently by Kutta [1] in 1902 and Joukowski [2] in 1906 in the case of a two-dimensional steady inviscid flow. This formula, called the Kutta-Joukowski theorem, already evidenced 


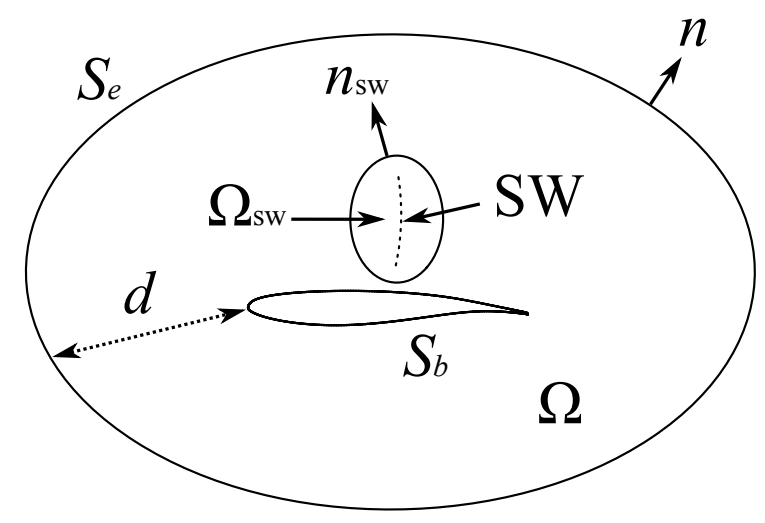

Fig. 1 Fluid domains and boundaries

the importance of vortices in the generation of the aerodynamic force since it related the lift to the circulation created by an airfoil. Nevertheless, it provided no drag: this is the d'Alembert's paradox. Moreover, no circulation can be generated in such a flow according to Kelvin's theorem, which explains the reluctance of the scientific community at that time. Yet, Bryant and Williams [3] later confirmed that the formula is also applicable to viscous flows. The paradox was lifted by Prandtl who introduced the concept of effectively inviscid flow [4], for which the fluid viscosity $\mu \rightarrow 0$ but $\mu \neq 0$. With this model, the circulation is set by the Kutta condition which accounts for the viscous effects in the boundary layer and states that the flow leaves the airfoil at the trailing edge. Prandtl also elaborated the Kutta-Joukowski formula upon adding a vertical velocity perturbation induced by trailing vortices. It allowed him to define the lift-induced drag, present only in three-dimensional flows around wings of finite span. Very recently, Schmitz [5] went further and identified a finite-domain correction term to the Kutta-Joukowski theorem due to viscous effects in the wake. In parallel with Prandtl's developments, Filon [6] investigated the drag experienced by a cylinder in an incompressible viscous flow and derived the analogue for drag to the Kutta-Joukowski theorem (see also Drela [7] p.245-250). Nevertheless, the aforementioned developments were limited to the study of incompressible flows. Using a small perturbation approximation, Liu et al. [8, 9] recently extended the use of Kutta-Joukowski and Filon's formulas to compressible viscous flows, but their testable force formula holds only in the linear far field.

Prior to the blooming of computational fluid dynamics (CFD), wind-tunnel testing was the only way to assess the aerodynamic performance of an aircraft (notwithstanding real flight tests). Therefore, numerous formulations were developed to extract the drag and decompose it from wake-plane data (see [10-12] for instance). Betz [13] and Taylor [14] both defined the profile drag (friction drag plus viscous pressure drag plus wave drag) as the total pressure loss in incompressible flows. Jones [15] later assumed more restrictive conditions and derived a profile drag formula also applicable to compressible flows [12]. A great leap forward was made with Maskell's lift-induced drag formula [16] valid in incompressible flows, although often used in compressible regime and widely used today in wind-tunnel measurements and CFD postprocessing tools [17--24]. However, those methods were all developed upon making approximations, which reduced their scope of application and inevitably led to inaccuracies in drag predictions.

The research on more accurate far-field formulations valid in compressible flows led to the development of thermodynamic approaches [25-29] now used in industrial applications and based on the entropy production within the boundary layers and across shock waves. Those formulas generalize Oswatitsch's profile drag definition [30, 31] and decompose it into viscous drag (friction drag plus viscous pressure drag) and wave drag. Unfortunately, the lift-induced drag is deduced by subtracting the profile drag from the total drag which highlights the lack of a phenomenological definition of this drag component in complex flows [32].

More recently, Schmitz and Coder [33, 34] developed an aerodynamic force prediction and breakdown based on partial pressure fields. This approach allowed them to decompose the lift and the drag into an inviscid and a dissipative component. In transonic flows, the lift-induced drag and the wave drag are both contained in the inviscid component. The same authors [35] later managed to decouple the wave drag generated by a weak shock from the lift-induced drag in inviscid flows.

The vortex-force theory belongs to the vorticity-based formulations (see [36] for a thorough review) which form another family of the far-field methods. This formula has aroused a great enthusiasm in the very recent years since it defines the lift and the lift-induced drag in complex viscous flows. It follows Prandtl's developments on the vortex 
force [4] and his famous inviscid lifting-line theory. Saffman [37] later studied this formulation in the case of an incompressible steady inviscid flow. In their derivations the pressure was eliminated by applying the Bernoulli equation and the aerodynamic force was solely expressed with the Lamb vector field $\boldsymbol{l}=\boldsymbol{\omega} \times \boldsymbol{q}$ where $\boldsymbol{\omega}=\nabla \times \boldsymbol{q}$ is the vorticity. The extension to the relevant case of the body wake convected infinitely downstream also including the steady case was conducted by Wu et al. [38, 39] where the pressure field was eliminated by using the derivative moment transformation (DMT) Eq. A.2):

$$
\boldsymbol{F}=-\rho_{\infty} \int_{\Omega} \boldsymbol{l} \mathrm{d} v-\frac{\rho_{\infty}}{\mathcal{N}-1} \oint_{S_{e}} \boldsymbol{r} \times(\boldsymbol{n} \times \boldsymbol{l}) \mathrm{d} S+\frac{1}{\mathcal{N}-1} \oint_{S_{e}} \boldsymbol{r} \times(\boldsymbol{n} \times \nabla \cdot \boldsymbol{\tau}) \mathrm{d} S+\oint_{S_{e}} \boldsymbol{\tau} \cdot \boldsymbol{n} \mathrm{d} S
$$

where $\mathcal{N}=2,3$ is the dimension of the physical space and $\boldsymbol{r}$ is the position vector. A compressible version of the preceding formulation was suggested by Wu et al. [38] (p. 621) and by Liu et al. [40, 41] who defined a compressible Lamb vector $\rho \boldsymbol{l}-\frac{q^{2}}{2} \nabla \rho$ such that

$$
\boldsymbol{F}=-\int_{\Omega}\left(\rho \boldsymbol{l}-\frac{q^{2}}{2} \nabla \rho\right) \mathrm{d} v-\frac{1}{\mathcal{N}-1} \oint_{S_{e}} \boldsymbol{r} \times\left[\boldsymbol{n} \times\left(\rho \boldsymbol{l}-\frac{q^{2}}{2} \nabla \rho\right)\right] \mathrm{d} S+\frac{1}{\mathcal{N}-1} \oint_{S_{e}} \boldsymbol{r} \times(\boldsymbol{n} \times \nabla \cdot \boldsymbol{\tau}) \mathrm{d} S+\oint_{S_{e}} \boldsymbol{\tau} \cdot \boldsymbol{n} \mathrm{d} S
$$

In incompressible flows, the integration is limited to the regions where the vorticity is nonzero since it is solely based on the Lamb vector field [42]. The case of compressible flows involves the additional term $\frac{q^{2}}{2} \nabla \rho$ which greatly extends the integration domain. To address this problem, Mele and Tognaccini [43] used Eq. A.1] in order to express Eq. 3. as follows:

$$
\boldsymbol{F}=\boldsymbol{F}_{\rho l}+\boldsymbol{F}_{m_{\rho}}+\boldsymbol{F}_{S_{e}}+\boldsymbol{F}_{\tau}
$$

where

$$
\begin{aligned}
\boldsymbol{F}_{\rho l} & =-\int_{\Omega} \rho \boldsymbol{l} \mathrm{d} v \\
\boldsymbol{F}_{m_{\rho}} & =-\frac{1}{\mathcal{N}-1} \int_{\Omega} \boldsymbol{r} \times\left(\nabla \rho \times \nabla\left(\frac{q^{2}}{2}\right)\right) \mathrm{d} v=-\int_{\Omega} \boldsymbol{m}_{\rho} \mathrm{d} v \\
\boldsymbol{F}_{S_{e}} & =-\frac{1}{\mathcal{N}-1} \oint_{S_{e}} \boldsymbol{r} \times(\boldsymbol{n} \times \rho \boldsymbol{l}) \mathrm{d} S \\
\boldsymbol{F}_{\boldsymbol{\tau}} & =\frac{1}{\mathcal{N}-1} \oint_{S_{e}} \boldsymbol{r} \times(\boldsymbol{n} \times \nabla \cdot \boldsymbol{\tau}) \mathrm{d} S+\oint_{S_{e}} \boldsymbol{\tau} \cdot \boldsymbol{n} \mathrm{d} S
\end{aligned}
$$

$\boldsymbol{F}_{\rho l}$ is the vortex force and provides the lift $L$ and the lift-induced drag $D_{i}$ in steady incompressible flows [44]; $\boldsymbol{F}_{m_{\rho}}$ is a compressibility term which contributes to $L$ and $D_{i}$ in steady compressible flows [43, 45]; $\boldsymbol{F}_{S_{e}}$ provides only a drag component [38, 43, 45]: the profile drag $D_{P}$. Besides, when $S_{e}$ retreats to infinity, the surface integration is performed in flow regions where viscous stresses are negligible such that $\boldsymbol{F}_{\tau}$ vanishes [44, 46]. In transonic flows however, Mele and Tognaccini [43] noticed that the expression of $\boldsymbol{F}_{m_{\rho}}$ given by Eq. (6) is unable to compute the correct contribution. Mele et al. [47] later explained that this is due to numerical inaccuracies in the shock wave region and used an alternative form of $\boldsymbol{F}_{m_{\rho}}$ which solved the problem. This alternative expression will be presented in the following.

The breakdown of the profile drag into viscous and wave contributions has also been investigated. The viscous drag is produced in the boundary layers while the wave drag is produced across shock waves, which makes it tough to distinguish them from one another since the corresponding wakes merge further downstream [47]. A first possibility consisted in defining the wave drag as the integration of $\boldsymbol{F}_{S_{e}}$ on a wake plane directly downstream of the shock. Later on, Ostieri and Tognaccini [48] asserted that this definition tends to systematically overestimate the wave drag. Rather, they transformed $\boldsymbol{F}_{S_{e}}$ into a volume integral in the boundary layer and shock wave regions $\Omega_{v}$ and $\Omega_{\mathrm{sw}}$. They noticed that the volume $\Omega_{\mathrm{sw}}$ must include the near wake of the shock for a better wave drag prediction [48]. Their definition was applied to two-dimensional symmetrical flows around the NACA0012 airfoil and still needs to be tested on more complex configurations.

Besides, a recurring issue related to far-field drag extraction and decomposition is the sensitivity of the drag breakdown to the location of the wake plane or, on a broader level, to the size of the control volume $\Omega$. Indeed, Zou et al. [49] investigated the effect of the physical and numerical dissipations on the lift-induced drag component and developed a formulation valid in incompressible flows which reduces the sensitivity of the decomposition. For their 
part, Kang et al. [50] decomposed the aerodynamic force into the reversible part $\boldsymbol{F}_{\rho l}+\boldsymbol{F}_{m_{\rho}}$ and the irreversible part $\boldsymbol{F}_{S_{e}}$ by applying the vortex-force theory to compressible inviscid flows. They also addressed the effect of the Reynolds number. Finally, they noticed that the drag breakdown is sensitive to the location of the reference point chosen for the computation of moment transformations.

Indeed, the position vector $\boldsymbol{r}$ is involved in the integral terms of $\boldsymbol{F}_{m_{\rho}}, \boldsymbol{F}_{S_{e}}$ and $\boldsymbol{F}_{\tau}$. Very recently, Fournis et al. [51,52] conducted a thorough study devoted to this sensitivity and developed an equivalent reference-point-invariant formulation based on far-field flow symmetries. The method was initially developed assuming that the external boundary $S_{e}$ is symmetrical with respect to the vertical and horizontal planes. Regarding the case of unsymmetrical surfaces, a symmetrization of the unit normal and the position vector must be performed but its implementation in algorithms can be tough.

As of now, the vortex-force theory constitutes in practice a serious alternative for predicting and decomposing the aerodynamic force into lift, lift-induced drag and profile drag. Moreover, in incompressible flows, several studies analyzed the physical role of each term by identifying the links between the vortex-force theory and classical far-field analyses: the Kutta-Joukowski theorem (see [44]), Maskell's formula (see [50]) and Betz's formula (see [38, 53]). In compressible flows however, a similar physical interpretation of the terms of the decomposition is still lacking. Moreover, it is desirable for an easier implementation to have at our disposal a naturally invariant formulation for which there is no need to consider the flow symmetries.

Hence, the goal of this paper is to develop a reference-point-invariant formulation of the vortex-force theory able to identify the role of the compressibility term $\boldsymbol{F}_{m_{\rho}}$ in lift and drag. This will be done by establishing for the first time the links between the vortex-force theory and the classical far-field analyses in transonic flows. The work is organized as follows: section IIII is a mathematical step which thoroughly handles the presence of discontinuities in the flow. It first presents a rigorous derivation of the vortex-force theory in transonic flows, where shock waves are modeled as discontinuities. Then, starting from this definition including the jump of flow quantities at the shock, $\boldsymbol{F}_{m_{\rho}}$ is re-expressed in its transonic version (first introduced by Mele et al. [47] but without considering any discontinuity). Hence, section IV establishes the link between the vortex-force theory and the classical far-field analyses leading to the definition of a new invariant formulation in transonic flows. In section $\mathrm{V}$, the new formulation is tested on the NASA CRM and the profile drag defined by Betz's formula is analyzed in compressible flows.

\section{Derivation of the vortex-force theory in the presence of shock waves}

In this section, the vortex-force theory is derived for the first time in the presence of shock discontinuities and an additional term of the decomposition involving the jump of flow quantities is identified. A re-expression of this additional term will later lead to the transonic expression of $\boldsymbol{F}_{m_{\rho}}$ first introduced by Mele et al. [47] in transonic flows.

\section{A. Application of the Lamb-vector-based momentum balance to a discontinuous domain}

In transonic flows, the fluid acceleration on the suction side of an airfoil may be such that a shock wave is formed. In practice, a shock wave is a layer which thickness has the same order of magnitude as the mean free path of the gas molecules. Since the Navier-Stokes equations are derived from the equations of motion of continuum mechanics, they cannot predict the internal structure of shock waves (see e.g. [31] p. 550-551 or [54] p. 353). That is why it is common to model the shock wave with a surface or line of discontinuity [31, 53,-55] denoted SW in Fig 2.

The transonic formulation is derived by proceeding to the same theoretical developments that led to its subsonic version Eq. (4) (see the derivations in [38, 39, 43]), but instead of the standard DMT Eqs. (A.1] and [A.2], one must use the generalized DMT Eqs. A.3 and (A.4). The steady Navier-Stokes equations write

$$
\nabla \cdot(\rho \boldsymbol{q} \boldsymbol{q})=\rho \boldsymbol{l}+\rho \nabla\left(\frac{q^{2}}{2}\right)=-\nabla p+\nabla \cdot \boldsymbol{\tau}
$$

The steady Helmholtz equations are found by applying the curl operator to the right-hand side of the last relation:

$$
\nabla \times(\rho l)+\nabla \rho \times \nabla\left(\frac{q^{2}}{2}\right)=\nabla \times(\nabla \cdot \tau)
$$

Splitting the fluid domain $\Omega=\Omega_{1} \cup \Omega_{2} \cup \Omega_{3}$ as shown in Fig 2 and using Eqs. A.3. and A.4, the Lamb-vector-based 


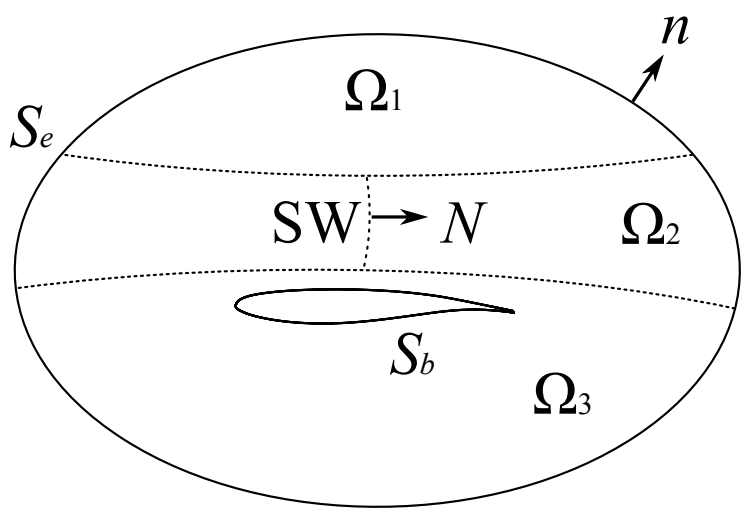

Fig. 2 Partition of the fluid domain in transonic flows

momentum balance in each sub-volume yields

$$
\begin{array}{ll}
\text { in } \Omega_{1} & \boldsymbol{F}_{\rho l_{1}}+\boldsymbol{F}_{m_{\rho_{1}}}+\boldsymbol{F}_{S_{e 1}}+\boldsymbol{F}_{\tau_{1}}=\mathbf{0} \\
\text { in } \Omega_{2} & \boldsymbol{F}_{\rho l_{2}}+\boldsymbol{F}_{m_{\rho_{2}}}+\boldsymbol{F}_{S_{e_{2}}}+\boldsymbol{F}_{\tau_{2}}=\int_{\mathrm{SW}} \llbracket \boldsymbol{\tau} \rrbracket \cdot \boldsymbol{N} \mathrm{d} S+\frac{1}{\mathcal{N}-1} \int_{\mathrm{SW}} \boldsymbol{r} \times\left(\boldsymbol{N} \times \llbracket \nabla p+\rho \nabla\left(\frac{q^{2}}{2}\right) \rrbracket\right) \mathrm{d} S \\
\text { in } \Omega_{3} & \boldsymbol{F}_{\rho l_{3}}+\boldsymbol{F}_{m_{\rho_{3}}}+\boldsymbol{F}_{S_{e 3}}+\boldsymbol{F}_{\tau_{3}}=-\oint_{S_{b}}(-p \boldsymbol{I}+\boldsymbol{\tau}) \cdot \boldsymbol{n d} S=\boldsymbol{F}
\end{array}
$$

where $\llbracket \bullet \rrbracket=\bullet_{d}-\bullet_{u}$ is the jump operator $\left(\bullet_{u}\right.$ is the upstream quantity and $\bullet_{d}$ is the downstream quantity) and $\boldsymbol{N}$ is the normal to the shock pointing in the downstream direction. Therefore, by adding Eqs.(11), (12) and (13) the transonic version of Eq. (4) is established:

$$
\boldsymbol{F}=\boldsymbol{F}_{\rho l}+\boldsymbol{F}_{m_{\rho}}+\boldsymbol{F}_{S_{e}}+\boldsymbol{F}_{\tau}+\boldsymbol{F}_{\mathrm{SW}}
$$

where

$$
\boldsymbol{F}_{\mathrm{SW}}=-\int_{\mathrm{SW}} \llbracket \boldsymbol{\tau} \rrbracket \cdot \boldsymbol{N} \mathrm{d} S-\frac{1}{\mathcal{N}-1} \int_{\mathrm{SW}} \boldsymbol{r} \times\left(\boldsymbol{N} \times \llbracket \nabla p+\rho \nabla\left(\frac{q^{2}}{2}\right) \rrbracket\right) \mathrm{d} S
$$

Hence the presence of the shock discontinuity implies the existence of an additional contribution $\boldsymbol{F}_{\text {SW }}$ which contains the jump of flow quantities at the shock. This term has hitherto never been noticed and its omission may well explain why Mele-Tognaccini's formulation [43] (where $\boldsymbol{F}_{m_{\rho}}$ is defined by Eq. 6) is not suited for transonic test cases. Yet, $\boldsymbol{F}_{\mathrm{SW}}$ is tough to assess in practice since the numerical scheme is only first order accurate in the shock wave region. Moreover, in the shock region, the derivatives of the flow quantities are no longer defined in the classical sense but rather in the sense of distributions, involving a jump across SW. The trick is then to find an equivalent form of $\boldsymbol{F}_{\text {SW }}$ avoiding the integration on the shock discontinuity. The next subsection will present the developments leading to this equivalent expression which, when added to the other force contributions, leads to Mele et al.'s formulation in transonic flows [47].

\section{B. Rigorous proof of Mele et al.'s formulation in transonic flows}

In this subsection, the transonic expression of $\boldsymbol{F}_{m_{\rho}}$ first introduced by Mele et al. [47] will be denoted $\boldsymbol{F}_{m_{\rho}}^{\text {trans }}$ and will be found by summing $\boldsymbol{F}_{m_{\rho}}$ and $\boldsymbol{F}_{\mathrm{SW}}$. Starting from the definition Eq. $15, \boldsymbol{F}_{\mathrm{SW}}$ is first re-expressed in order to avoid the integration on the shock discontinuity (see Appendix $B$. The new expression Eq. $B .6$ ) of $\boldsymbol{F}_{\mathrm{SW}}$ does not imply integrations on SW and is therefore easily assessable in practice when dealing with CFD computations. Although $\boldsymbol{F}_{\mathrm{SW}}$ can be let in this form at first glance, it is more convenient to merge it with $\boldsymbol{F}_{m_{\rho}}$ :

$$
\begin{aligned}
\boldsymbol{F}_{m_{\rho}}+\boldsymbol{F}_{\mathrm{SW}}= & -\int_{\Omega \backslash \Omega_{\mathrm{sw}}} \boldsymbol{m}_{\rho} \mathrm{d} v+\oint_{\partial \Omega_{\mathrm{sw}}}\left(\rho \frac{q^{2}}{2} \boldsymbol{n}_{\mathrm{sw}}-\rho \boldsymbol{q}\left(\boldsymbol{q} \cdot \boldsymbol{n}_{\mathrm{sW}}\right)\right) \mathrm{d} S \\
& +\frac{1}{\mathcal{N}-1} \oint_{\partial \Omega_{\mathrm{sw}}} \boldsymbol{r} \times\left(\boldsymbol{n}_{\mathrm{sw}} \times \frac{q^{2}}{2} \nabla \rho\right) \mathrm{d} S+\int_{\Omega_{\mathrm{sw}}} \rho \boldsymbol{l} \mathrm{d} v
\end{aligned}
$$


where $\boldsymbol{n}_{\mathrm{sw}}$ denotes the unit normal pointing outside $\Omega_{\mathrm{sw}}$. The right-hand side of Eq. 16 is nothing but the transonic expression $\boldsymbol{F}_{m_{\rho}}^{\text {trans }}$ of the term $\boldsymbol{F}_{m_{\rho}}$ first introduced by Mele et al. [47]:

$$
\boldsymbol{F}_{m_{\rho}}+\boldsymbol{F}_{\mathrm{SW}}=\boldsymbol{F}_{m_{\rho}}^{\mathrm{trans}}
$$

In the end, this cumbersome mathematical step leading to Eq.(17) is necessary since it provides a more rigorous derivation of $\boldsymbol{F}_{m_{\rho}}^{\text {trans }}$, and hence of Mele et al.'s transonic formulation. Besides, Mele et al. [47] asserted that the errors occuring near the shock wave are imputable exclusively to the altered accuracy of the numerical scheme in this region. This time, by considering the discontinuities of the flow, the presence of $\boldsymbol{F}_{\mathrm{SW}}$ in Eq. 17) illustrates in mathematical terms (rather than in numerical terms) why Mele-Tognaccini's formulation [43] is incomplete when dealing with shock waves. Hence, the aerodynamic force summarizes as

$$
\boldsymbol{F}=\boldsymbol{F}_{\rho l}+\boldsymbol{F}_{m_{\rho}}^{\text {trans }}+\boldsymbol{F}_{S_{e}}+\boldsymbol{F}_{\tau}
$$

and can now be applied to transonic test cases [47]. The next section will present how the force decomposition in Eq. 18 is related to the Kutta-Joukowski theorem and Maskell's formula through the term $\boldsymbol{F}_{\rho l}+\boldsymbol{F}_{m_{\rho}}^{\text {trans }}$ and to Betz's formula through the term $\boldsymbol{F}_{S_{e}}+\boldsymbol{F}_{\tau}$. Finally, a new formulation will be proposed and analyzed.

\section{Development of an invariant formulation embedding classical far-field theories}

In this section, further theoretical developments enable to identify the role of $\boldsymbol{F}_{m_{\rho}}^{\text {trans }}$ in lift and drag by summarizing, for the first time in a single equation, the relation between the vortex-force theory and classical far-field analyses in transonic flows. Later, starting from this relation, a new reference-point-invariant decomposition is defined.

\section{A. Links with the Kutta-Joukowski lift theorem and Maskell's lift-induced drag formula}

Previous works already addressed in mathematical terms the links between $\boldsymbol{F}_{\rho l}$, the Kutta-Joukowski lift theorem and Maskell's lift-induced drag formula [44, 50] in incompressible flows. In compressible flows however, it was shown through numerical simulations only that the relative importance of $\boldsymbol{F}_{\rho l}$ in the total lift decreases in favour of $\boldsymbol{F}_{m_{\rho}}$ when increasing the Mach number [43, 45, 47]: it supports former observations made by Chang et al. [56, 57]. It was also shown that $\boldsymbol{F}_{m_{\rho}}$ plays an essential role in the lift-induced drag as well [43, 45]. Very recently, a theoretical study was conducted to effectively prove that $\boldsymbol{F}_{m_{\rho}}$ contributes to the lift and the lift-induced drag in subsonic flows [58]. It consisted in finding the links between $\boldsymbol{F}_{\rho l}+\boldsymbol{F}_{m_{\rho}}$, the Kutta-Joukowski theorem and Maskell's formula in compressible flows.

The same analysis is now carried out in transonic flows with $\boldsymbol{F}_{m_{\rho}}^{\text {trans }}$. To do so, the first volume integral of Eq. 16 is re-expressed using the first standard DMT Eq.A.1):

$$
-\int_{\Omega \backslash \Omega_{\mathrm{sw}}} \boldsymbol{m}_{\rho} \mathrm{d} v=\int_{\Omega \backslash \Omega_{\mathrm{sw}}} \frac{q^{2}}{2} \nabla \rho \mathrm{d} v+\frac{1}{\mathcal{N}-1} \oint_{S_{e}} \boldsymbol{r} \times\left(\boldsymbol{n} \times \frac{q^{2}}{2} \nabla \rho\right) \mathrm{d} S-\frac{1}{\mathcal{N}-1} \oint_{\partial \Omega_{\mathrm{sw}}} \boldsymbol{r} \times\left(\boldsymbol{n}_{\mathrm{sw}} \times \frac{q^{2}}{2} \nabla \rho\right) \mathrm{d} S
$$

By using Eq.17], and with the introduction of Eq.19] into Eq.16, $\boldsymbol{F}_{m_{\rho}}^{\text {trans }}$ can be expressed as follows:

$$
\begin{aligned}
\boldsymbol{F}_{m_{\rho}}^{\mathrm{trans}}= & \int_{\Omega \backslash \Omega_{\mathrm{sw}}} \frac{q^{2}}{2} \nabla \rho \mathrm{d} v+\frac{1}{\mathcal{N}-1} \oint_{S_{e}} \boldsymbol{r} \times\left(\boldsymbol{n} \times \frac{q^{2}}{2} \nabla \rho\right) \mathrm{d} S \\
& +\oint_{\partial \Omega_{\mathrm{sw}}}\left(\rho \frac{q^{2}}{2} \boldsymbol{n}_{\mathrm{sw}}-\rho \boldsymbol{q}\left(\boldsymbol{q} \cdot \boldsymbol{n}_{\mathrm{sw}}\right)\right) \mathrm{d} S+\int_{\Omega_{\mathrm{sw}}} \rho \boldsymbol{l} \mathrm{d} v
\end{aligned}
$$

When adding the contribution of $\boldsymbol{F}_{\rho l}$ one obtains

$$
\begin{aligned}
\boldsymbol{F}_{\rho l}+\boldsymbol{F}_{m_{\rho}}^{\mathrm{trans}}= & -\int_{\Omega \backslash \Omega_{\mathrm{sw}}}\left(\rho \boldsymbol{l}-\frac{q^{2}}{2} \nabla \rho\right) \mathrm{d} v+\frac{1}{\mathcal{N}-1} \oint_{S_{e}} \boldsymbol{r} \times\left(\boldsymbol{n} \times \frac{q^{2}}{2} \nabla \rho\right) \mathrm{d} S \\
& +\oint_{\partial \Omega_{\mathrm{sw}}}\left(\rho \frac{q^{2}}{2} \boldsymbol{n}_{\mathrm{sw}}-\rho \boldsymbol{q}\left(\boldsymbol{q} \cdot \boldsymbol{n}_{\mathrm{sw}}\right)\right) \mathrm{d} S
\end{aligned}
$$


Using the Navier-Stokes equations Eq. 9 , it is possible to show that

$$
\rho \boldsymbol{l}-\frac{q^{2}}{2} \nabla \rho=\nabla \cdot\left(\rho \boldsymbol{q} \boldsymbol{q}-\rho \frac{q^{2}}{2} \boldsymbol{I}\right)
$$

and the volume integral in Eq. 21] is re-expressed using the divergence theorem:

$$
-\int_{\Omega \backslash \Omega_{\mathrm{sw}}}\left(\rho \boldsymbol{l}-\frac{q^{2}}{2} \nabla \rho\right) \mathrm{d} v=\oint_{S_{e}}\left(\rho \frac{q^{2}}{2} \boldsymbol{n}-\rho \boldsymbol{q}(\boldsymbol{q} \cdot \boldsymbol{n})\right) \mathrm{d} S-\oint_{\partial \Omega_{\mathrm{sw}}}\left(\rho \frac{q^{2}}{2} \boldsymbol{n}_{\mathrm{sw}}-\rho \boldsymbol{q}\left(\boldsymbol{q} \cdot \boldsymbol{n}_{\mathrm{sw}}\right)\right) \mathrm{d} S
$$

such that

$$
\boldsymbol{F}_{\rho l}+\boldsymbol{F}_{m_{\rho}}^{\text {trans }}=\oint_{S_{e}}\left(\rho \frac{q^{2}}{2} \boldsymbol{n}-\rho \boldsymbol{q}(\boldsymbol{q} \cdot \boldsymbol{n})\right) \mathrm{d} S+\frac{1}{\mathcal{N}-1} \oint_{S_{e}} \boldsymbol{r} \times\left(\boldsymbol{n} \times \frac{q^{2}}{2} \nabla \rho\right) \mathrm{d} S
$$

Hence, $\boldsymbol{F}_{\rho l}+\boldsymbol{F}_{m_{\rho}}^{\text {trans }}$ is solely expressed with surface integrals on the external boundary $S_{e}$. The velocity vector is decomposed as $\boldsymbol{q}=\boldsymbol{U}_{\infty}+\delta \boldsymbol{q}$. Using the steady continuity equation

$$
\oint_{S_{e}} \rho \boldsymbol{q} \cdot \boldsymbol{n} \mathrm{d} S=0
$$

Eq. 23] becomes [58]

$$
\begin{aligned}
\boldsymbol{F}_{\rho l}+\boldsymbol{F}_{m_{\rho}}^{\mathrm{trans}}= & \boldsymbol{U}_{\infty} \times \oint_{S_{e}} \boldsymbol{n} \times \rho \delta \boldsymbol{q} \mathrm{d} S \\
& +\oint_{S_{e}} \rho \frac{\delta q^{2}}{2} \boldsymbol{n} \mathrm{d} S-\oint_{S_{e}} \rho \delta \boldsymbol{q}(\delta \boldsymbol{q} \cdot \boldsymbol{n}) \mathrm{d} S \\
& +\frac{U_{\infty}^{2}}{2} \oint_{S_{e}}\left(\rho-\rho_{\infty}\right) \boldsymbol{n} \mathrm{d} S+\frac{1}{\mathcal{N}-1} \oint_{S_{e}} \boldsymbol{r} \times\left(\boldsymbol{n} \times \frac{q^{2}}{2} \nabla \rho\right) \mathrm{d} S
\end{aligned}
$$

The first line of Eq. 24) resembles the Kutta-Joukowski theorem in compressible flows and contributes solely to the lift. The second line contributes both to the lift and the drag: its lift contribution is a correction to the Kutta-Joukowski formula and its drag component is the lift-induced drag. Finally the third line is a compressibility correction $\boldsymbol{F}_{\nabla} \rho$ vanishing in the wake [58]. $\boldsymbol{F}_{\rho l}+\boldsymbol{F}_{m_{\rho}}^{\text {trans }}$ is then expressed as follows:

$$
\boldsymbol{F}_{\rho l}+\boldsymbol{F}_{m_{\rho}}^{\text {trans }}=\boldsymbol{F}_{\mathrm{KJ}}+\boldsymbol{F}_{\mathrm{MSK}}+\boldsymbol{F}_{\nabla \rho}
$$

with

$$
\begin{aligned}
\boldsymbol{F}_{\mathrm{KJ}} & =\boldsymbol{U}_{\infty} \times \oint_{S_{e}} \boldsymbol{n} \times \rho \delta \boldsymbol{q} \mathrm{d} S+\oint_{S_{e}} \rho\left(\frac{\delta q^{2}}{2} n_{z}-w(\delta \boldsymbol{q} \cdot \boldsymbol{n})\right) \mathrm{d} S \boldsymbol{e}_{z} \\
\boldsymbol{F}_{\mathrm{MSK}} & =\oint_{S_{e}} \rho\left(\frac{\delta q^{2}}{2} n_{x}-u(\delta \boldsymbol{q} \cdot \boldsymbol{n})\right) \mathrm{d} S \boldsymbol{e}_{x} \\
\boldsymbol{F}_{\nabla \rho} & =\frac{U_{\infty}^{2}}{2} \oint_{S_{e}}\left(\rho-\rho_{\infty}\right) \boldsymbol{n} \mathrm{d} S+\frac{1}{\mathcal{N}-1} \oint_{S_{e}} \boldsymbol{r} \times\left(\boldsymbol{n} \times \frac{q^{2}}{2} \nabla \rho\right) \mathrm{d} S
\end{aligned}
$$

Here, $\boldsymbol{F}_{\mathrm{KJ}}$ is a generalized Kutta-Joukowski theorem and $\boldsymbol{F}_{\mathrm{MSK}}$ is an extension of Maskell's formula. Besides, the velocity perturbation $\delta \boldsymbol{q}$ is nonzero only in the wake provided that $S_{e}$ is not too close to the aircraft. Therefore, integrals involving $\delta \boldsymbol{q}$ can be limited to a wake plane $W$ on which $\boldsymbol{n}=\boldsymbol{e}_{x}$ (see also [44]). By projecting onto the $x$ and $z$ axes, $\boldsymbol{F}_{\mathrm{KJ}}$ and $\boldsymbol{F}_{\mathrm{MSK}}$ reduce to more familiar expressions:

$$
\begin{aligned}
\boldsymbol{F}_{\mathrm{KJ}} & =-\rho_{\infty} U_{\infty}\left(\Gamma^{*}+\delta \Gamma^{*}\right) \boldsymbol{e}_{z} \\
\boldsymbol{F}_{\mathrm{MSK}} & =\frac{1}{2} \int_{W} \rho\left(v^{2}+w^{2}-u^{2}\right) \mathrm{d} S \boldsymbol{e}_{x}
\end{aligned}
$$

where

$$
\boldsymbol{\Gamma}^{*}=\oint_{S_{e}} \boldsymbol{n} \times \frac{\rho}{\rho_{\infty}} \delta \boldsymbol{q} \mathrm{d} S=-\Gamma^{*} \boldsymbol{e}_{y}
$$


is a circulation in compressible flows and

$$
\delta \Gamma^{*}=\frac{1}{U_{\infty}} \int_{W} \frac{\rho}{\rho_{\infty}} u w \mathrm{~d} S
$$

is a finite-domain viscous correction to $\Gamma^{*}$, first identified by Schmitz [5] in incompressible flows (without $\rho / \rho_{\infty}$ ). $\boldsymbol{F}_{\text {MSK }}$ provides a drag contribution only, the lift-induced drag, and is a compressible extension of the vortex-drag formula derived by Maskell in incompressible flows [16]. He asserted that the contribution of $-u^{2}$ (later analyzed by Spalart [32]) is small and should be assigned to the profile drag. In this study, this term is however kept in the lift-induced drag contribution.

In the end it was shown that, in transonic flows, $\boldsymbol{F}_{\rho l}+\boldsymbol{F}_{m_{\rho}}^{\text {trans }}$ differs from the classical Kutta-Joukowski theorem and Maskell's formula only by a compressibility correction $\boldsymbol{F}_{\nabla \rho}$ which involves density gradients and variations. As $S_{e}$ extends further away from the body surface, this correction gradually vanishes while $\boldsymbol{F}_{\mathrm{KJ}}$ and $\boldsymbol{F}_{\text {MSK }}$ respectively reduce to the incompressible Kutta-Joukowski theorem (see [5]) and the incompressible Maskell's formula (see [16-19, 59]). As it happens, it was shown by several authors that the incompressible Kutta-Joukowski theorem can predict the lift in compressible flows [8, 9, 43]. Moreover, the incompressible Maskell's formula can also be used for evaluating the lift-induced drag in compressible regime [17,-19, 22], provided that the integration is performed far enough in the wake. Hence, the preceding theoretical analysis clearly evidences that $\boldsymbol{F}_{m_{\rho}}^{\text {trans }}$ plays an essential role in the lift and the lift-induced drag in transonic flows, so it must be added to $\boldsymbol{F}_{\rho l}$ in their definition. In the next subsection, it will be shown that the vortex-force theory is also closely related to the works of Betz on the profile drag [13].

\section{B. Link with Betz's profile drag formula}

In incompressible flows, $\boldsymbol{F}_{S_{e}}+\boldsymbol{F}_{\tau}$ was identified to be equal to the surface integral of the total pressure $p_{t}=p+\frac{1}{2} \rho_{\infty} q^{2}$ on $S_{e}$ [38, 50, 53]. In 1925, Betz [13] considered a non-lifting incompressible flow around an airfoil such that $v=w=0$, and for which the total drag is equal to the profile drag. He defined the profile drag as follows:

$$
D_{P}=\int_{W}\left(p_{t \infty}-p_{t}\right) \mathrm{d} S+\frac{1}{2} \rho_{\infty} \int_{W}\left(u^{\prime}-u\right)\left(u^{\prime}+u\right) \mathrm{d} S
$$

where $p_{t \infty}=p_{\infty}+\frac{1}{2} \rho_{\infty} U_{\infty}^{2}$ and $u^{\prime}$ is such that $p+\frac{1}{2} \rho_{\infty}\left(U_{\infty}+u^{\prime}\right)^{2}=p_{t \infty}$. With a quantitative analysis, he concluded that the second integral of Eq.31] has a contribution of less than 1/20 of the first and is therefore negligible. Hence, Betz defined the profile drag as the total pressure loss past a body. In compressible flows however, this definition does not hold anymore as already noticed by Kang et al. [50].

In this subsection, it is shown that the contribution of $\boldsymbol{F}_{S_{e}}+\boldsymbol{F}_{\tau}$ consists of the total pressure loss first introduced by Betz plus a nonvanishing compressibility term accounting for density variations on $S_{e}$. The Navier-Stokes equations are written as

$$
\rho \boldsymbol{l}-\nabla \cdot \boldsymbol{\tau}=-\nabla p_{t}+\frac{q^{2}}{2} \nabla \rho
$$

and starting from Eqs. (7) and (8), $\boldsymbol{F}_{S_{e}}+\boldsymbol{F}_{\tau}$ is expressed as follows:

$$
\begin{aligned}
\boldsymbol{F}_{S_{e}}+\boldsymbol{F}_{\tau}= & \oint_{S_{e}} \boldsymbol{\tau} \cdot \boldsymbol{n} \mathrm{d} S+\frac{1}{\mathcal{N}-1} \oint_{S_{e}} \boldsymbol{r} \times\left(\boldsymbol{n} \times \nabla p_{t}\right) \mathrm{d} S \\
& -\frac{1}{\mathcal{N}-1} \oint_{S_{e}} \boldsymbol{r} \times\left(\boldsymbol{n} \times \frac{q^{2}}{2} \nabla \rho\right) \mathrm{d} S
\end{aligned}
$$

The second integral of Eq. 32 is simplified using the second standard DMT Eq. A.2 (no discontinuity crosses $S_{e}$ ) and by using the definition of $\boldsymbol{F}_{\nabla \rho}$ in Eq. 28, it is possible to write

$$
\boldsymbol{F}_{S_{e}}+\boldsymbol{F}_{\tau}=\boldsymbol{F}_{\mathrm{BETZ}}-\boldsymbol{F}_{\nabla \rho}
$$

with

$$
\boldsymbol{F}_{\mathrm{BETZ}}=\oint_{S_{e}} \boldsymbol{\tau} \cdot \boldsymbol{n} \mathrm{d} S+\oint_{S_{e}}\left(p_{t \infty}-p_{t}\right) \boldsymbol{n} \mathrm{d} S+\frac{U_{\infty}^{2}}{2} \oint_{S_{e}}\left(\rho-\rho_{\infty}\right) \boldsymbol{n} \mathrm{d} S
$$

Hence $\boldsymbol{F}_{S_{e}}+\boldsymbol{F}_{\tau}$ is related to a compressible Betz's formula accounting for the total pressure loss plus the change in dynamic pressure due to density variations of a fluid flowing at velocity $U_{\infty}$. As mentioned earlier, $\boldsymbol{F}_{\nabla \rho}$ vanishes in the wake. 
In the end, it was shown that $\boldsymbol{F}_{S_{e}}+\boldsymbol{F}_{\tau}$ differs from an extension of Betz's formula by the same compressibility correction as before. Nevertheless, $\boldsymbol{F}_{\text {BETZ }}$ does not reduce to the incompressible Betz's formula when $S_{e}$ extends further away from $S_{b}$ : numerical simulations will show that the third integral of Eq. 34) does not vanish and that its lift contribution exactly counterbalances that of the second integral. The next subsection will now present a new formulation strictly equivalent to the classical far-field analyses and naturally independent of the reference point.

\section{Definition of the new formulation in transonic flows}

In 2017, Mele et al. [47] did not consider the term $\boldsymbol{F}_{\tau}$ since it is negligible in high-Reynolds-number flows when $S_{e}$ is far from $S_{b}$. They decomposed the aerodynamic force as follows:

$$
\begin{aligned}
& L^{\text {Mele }}=\boldsymbol{e}_{z} \cdot\left(\boldsymbol{F}_{\rho l}+\boldsymbol{F}_{m_{\rho}}^{\text {trans }}\right) \\
& D_{i}^{\text {Mele }}=\boldsymbol{e}_{x} \cdot\left(\boldsymbol{F}_{\rho l}+\boldsymbol{F}_{m_{\rho}}^{\text {trans }}\right) \\
& D_{P}^{\text {Mele }}=\boldsymbol{e}_{x} \cdot \boldsymbol{F}_{S_{e}}
\end{aligned}
$$

The aforementioned mathematical developments demonstrated that $\boldsymbol{F}_{\rho l}+\boldsymbol{F}_{m_{\rho}}^{\text {trans }}$ is linked to the Kutta-Joukowski theorem and Maskell's formula and that $\boldsymbol{F}_{S_{e}}\left(+\boldsymbol{F}_{\tau}\right)$ is linked to Betz's formula. However, the equivalence between Mele et al.'s decomposition and those classical analyses is exact only in the far field where the compressibility correction $\boldsymbol{F}_{\nabla \rho}$ is negligible. Unfortunately, the dissipation of the trailing vortices caused by the viscous diffusion and the numerical scheme in this region induces a transfer from the lift-induced drag to the profile drag [60] and alters the quality of the decomposition. Moreover, it was shown that this decomposition is sensitive to the choice of the reference point [50-52], which reduces its scope of application.

In order to better decompose the aerodynamic force when $S_{e}$ is close to the aircraft, a new formulation developed at ONERA and valid in transonic flows is proposed. Starting from Eqs. (18), 25) and (33), it is possible to show that the vortex-force theory encompasses the classical far-field analyses in a single expression:

$$
\boldsymbol{F}=\boldsymbol{F}_{\mathrm{KJ}}+\boldsymbol{F}_{\mathrm{MSK}}+\boldsymbol{F}_{\mathrm{BETZ}}
$$

with $\boldsymbol{F}_{\mathrm{KJ}}$ and $\boldsymbol{F}_{\mathrm{MSK}}$ respectively computed by Eqs. 26) and 27) in practice. Therefore, having that

$$
\begin{aligned}
\boldsymbol{F}_{\mathrm{KJ}}+\boldsymbol{F}_{\mathrm{MSK}} & =\boldsymbol{F}_{\rho l}+\boldsymbol{F}_{m_{\rho}}^{\text {trans }}-\boldsymbol{F}_{\nabla \rho} \\
\boldsymbol{F}_{\mathrm{BETZ}} & =\boldsymbol{F}_{S_{e}}+\boldsymbol{F}_{\tau}+\boldsymbol{F}_{\nabla \rho}
\end{aligned}
$$

the proposed ONERA decomposition is the following:

$$
\begin{aligned}
& L^{\mathrm{ONERA}}=\boldsymbol{e}_{z} \cdot\left(\boldsymbol{F}_{\rho l}+\boldsymbol{F}_{m_{\rho}}^{\mathrm{trans}}-\boldsymbol{F}_{\nabla \rho}\right) \\
& D_{i}^{\mathrm{ONERA}}=\boldsymbol{e}_{x} \cdot\left(\boldsymbol{F}_{\rho l}+\boldsymbol{F}_{m_{\rho}}^{\mathrm{trans}}-\boldsymbol{F}_{\nabla \rho}\right) \\
& D_{P}^{\mathrm{ONERA}}=\boldsymbol{e}_{x} \cdot\left(\boldsymbol{F}_{S_{e}}+\boldsymbol{F}_{\tau}+\boldsymbol{F}_{\nabla \rho}\right)
\end{aligned}
$$

Moreover, according to Eqs. (39) and [40], this new decomposition is actually equivalent to another brand new decomposition directly based on the classical far-field analyses and from now on referred to as the Kutta-JoukowskiMaskell-Betz (KJMB) decomposition:

$$
\begin{aligned}
L^{\mathrm{KJMB}} & =\boldsymbol{e}_{z} \cdot \boldsymbol{F}_{\mathrm{KJ}} \\
D_{i}^{\mathrm{KJMB}} & =\boldsymbol{e}_{x} \cdot \boldsymbol{F}_{\mathrm{MSK}} \\
D_{P}^{\mathrm{KJMB}} & =\boldsymbol{e}_{x} \cdot \boldsymbol{F}_{\mathrm{BETZ}}
\end{aligned}
$$

It is important to note that no approximation has been made in the derivation of the ONERA and KJMB formulations: the total force expression is an exact formula in each case. The ONERA formulation is strictly equivalent to the KJMB formulation and hence to the classical far-field analyses, no matter the location of $S_{e}$, which allows for an integration in regions where the grid resolution is still fine. More importantly, a key advantage is that the ONERA decomposition is naturally invariant to the reference point since $\boldsymbol{r}$ does not appear in $\boldsymbol{F}_{\mathrm{KJ}}, \boldsymbol{F}_{\mathrm{MSK}}$ and $\boldsymbol{F}_{\mathrm{BETZ}}$ (another proof is presented 
in Appendix C]. Finally, the Kutta-Joukowski theorem, Maskell's formula and Betz's formula can be interpreted in terms of local flow structures since the ONERA decomposition involves the Lamb vector (in $\boldsymbol{F}_{\rho l}$ and $\boldsymbol{F}_{S_{e}}$ ), the density gradient (in $\boldsymbol{F}_{m_{\rho}}^{\text {trans }}$ and $\boldsymbol{F}_{\nabla \rho}$ ) and other derivatives of the velocity vector (in $\boldsymbol{F}_{\tau}$ ).

In the following, the ONERA formulation will be compared through numerical computations to Mele et al.'s decomposition [47] summarized in Eqs. (35), (36) and (37) and to the thermodynamic approach of Destarac and Van der Vooren [28] (superscript VdV). The results provided by the KJMB formulation will also be presented. Finally, an in-depth study of $\boldsymbol{F}_{\text {BETZ }}$ will be conducted.

\section{Analysis of the results}

\section{A. Presentation of the test case}

The ONERA formulation was tested on a wing-fuselage configuration in order to show its potential for industrial purposes. A Reynolds-averaged-Navier-Stokes (RANS) simulation using Spalart-Allmaras turbulence model and Jameson's numerical scheme (cell-centered, $2^{\text {nd }}$ and $4^{\text {th }}$ order artificial viscosities) was performed in the AIRBUSSAFRAN-ONERA elsA solver [61] on the NASA CRM (see Fig 3] in transonic cruise flight conditions: $M_{\infty}=0.85$, $R e=5 \times 10^{6}$ and $C_{L} \approx 0.5$.

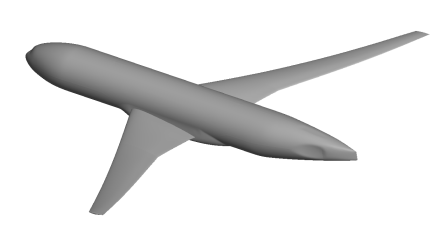

\section{Fig. 3 Geometry of the NASA CRM wing-fuselage configuration}

The mesh used in this study is the L4' version from the fifth Drag Prediction Workshop (DPW) [62] which is a fine mesh. It is a multiblock grid with 17,252,352 hexahedral cells. The $y+$ is between 0.5 and 1 and the far field lies more than 100 reference chord lengths away from the body. This mesh has exactly the same characteristics as the L4 version [63] but has been modified with a wing-twist to match wind-tunnel experiments. The reference chord of the wing is $c_{\text {ref }}=7.00532 \mathrm{~m}$. Convergence was attained and the residuals decreased by 13 orders of magnitude. A grid convergence study on this configuration can be found in Table 2 in Appendix D.

\section{B. Validation of the ONERA formulation}

In this subsection, the ONERA decomposition is compared to the equivalent KJMB decomposition and to Mele et al.'s decomposition [47]. In the KJMB formulation, it was shown that $\boldsymbol{F}_{\mathrm{KJ}}$ and $\boldsymbol{F}_{\mathrm{MSK}}$ can be limited to a wake plane $W$ perpendicular to $\boldsymbol{U}_{\infty}$. However, the two contributions are computed on $S_{e}$ (Eqs. (26) and (27)) in order to keep the exactness of the formula and to avoid any numerical errors introduced by the interpolation of the solution onto $W$. The evolutions of the various force decompositions with respect to the size of the integration domain are presented in Figs 4 and 5 . Here, the location of $S_{e}$ (hence the size of the integration domain) is changed by increasing the ratio $d / c_{\text {ref }}$ where $d$ is the distance between $S_{e}$ and $S_{b}$ as sketched in Fig 1 .

The evolution of the lift with respect to $d / c_{\text {ref }}$ is shown in Fig $4 \mathrm{a}$. The ONERA decomposition and Mele et al.'s decomposition only differ from one another by $\boldsymbol{F}_{\nabla \rho}$. Yet, they provide two distinct lift evolutions. In fact, $C_{L}^{\text {Mele }}$ decreases as $d / c_{\text {ref }}$ is increased whereas $C_{L}^{\text {ONERA }}$ increases. Eventually, both converge to the same value, confirming that the lift component of $\boldsymbol{F}_{\nabla \rho}$ disappears. The lift contribution $C_{L}^{\mathrm{KJMB}}$ provided by the KJMB formulation finally converges to the near-field lift $C_{L}^{\text {Near-field }}$. It confirms the capacity of the Kutta-Joukowski theorem to correctly compute the lift on an aircraft in transonic flight.

The prediction of the drag is given in Fig 4b. Given that the ONERA formulation and that of Mele et al. [47] provide the same total drag $\left(\boldsymbol{F}_{\tau}\right.$ is indeed negligible), only the value computed by the ONERA formulation is shown here. This value is always constant and equal to the near-field $\operatorname{drag} C_{D}^{\text {Near-field }}$ for $d / c_{\text {ref }} \geq 1$ and the tiny variations 
noticed for $d / c_{\text {ref }} \leq 1$ are imputable to numerical effects. Regarding the drag decomposition, it can be seen that the ONERA formulation always provides a greater lift-induced drag contribution than that computed by Mele et al.'s formulation. Conversely, the profile drag contribution is always lower, suggesting that the drag contribution of $\boldsymbol{F}_{\nabla \rho}$ is still not neglibible for $d / c_{\text {ref }} \leq 2$.

The evolution of the lift-induced drag is better grasped in Fig $5 \mathrm{a}$ where both formulations are compared to $C_{D_{i}}^{\mathrm{KJMB}}$ and $C_{D_{i}}^{\mathrm{VdV}}$, the prediction of Destarac and Van der Vooren's thermodynamic formulation [28]. The equivalence between the ONERA and KJMB formulations is confirmed and, compared to Mele et al.'s formulation, both yield a lift-induced drag contribution in better agreement with Destarac and Van der Vooren's prediction. In particular, $C_{D_{i}}^{\mathrm{VdV}}$ is 15 drag counts higher than $C_{D_{i}}^{\text {Mele }}$ and only 6 drag counts higher than $C_{D_{i}}^{\text {ONERA }}$ at $d / c_{\text {ref }}=1$ (see Table 1 . Moreover, $C_{D_{i}}^{\text {ONERA }}$

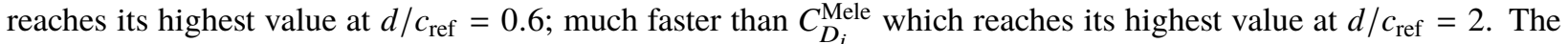
increase in lift-induced drag occuring for small values of $d / c_{\text {ref }}$ is mainly due to the contribution of the Lamb vector in the very near wake [44, 45, 50]. The increase is also due to the contribution of $\boldsymbol{F}_{\nabla \rho}$. Indeed, it is subtracted from the lift-induced drag definition of Mele et al. [47], and the evolution of $C_{D_{i}}^{\mathrm{ONERA}}$ is less sensitive to the size of the integration domain.

The evolution of the profile drag contribution is shown in Fig $5 b$. The ONERA and KJMB formulations yield a

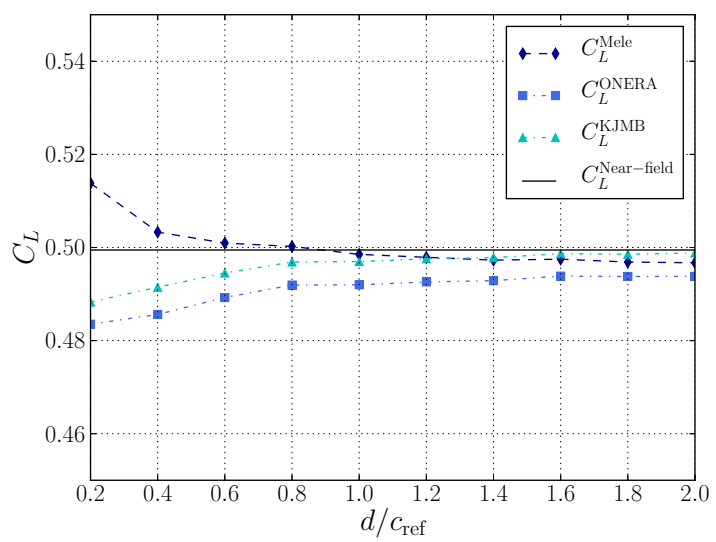

(a) Lift

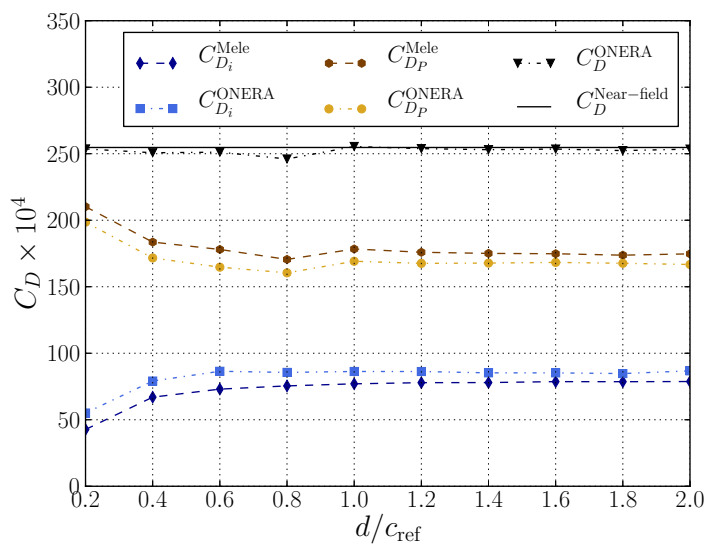

(b) Drag

Fig. 4 Lift and drag predictions on the NASA CRM, $M_{\infty}=0.85, R e=5 \times 10^{6}, C_{L} \approx 0.5$

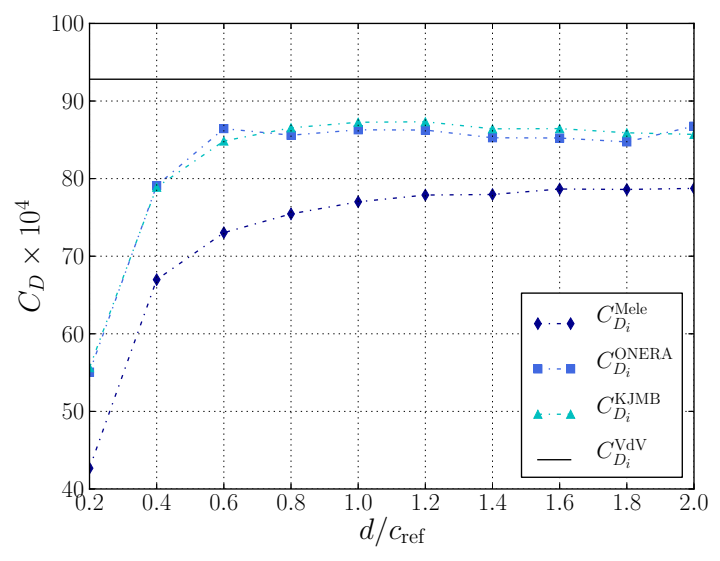

(a) Lift-induced drag

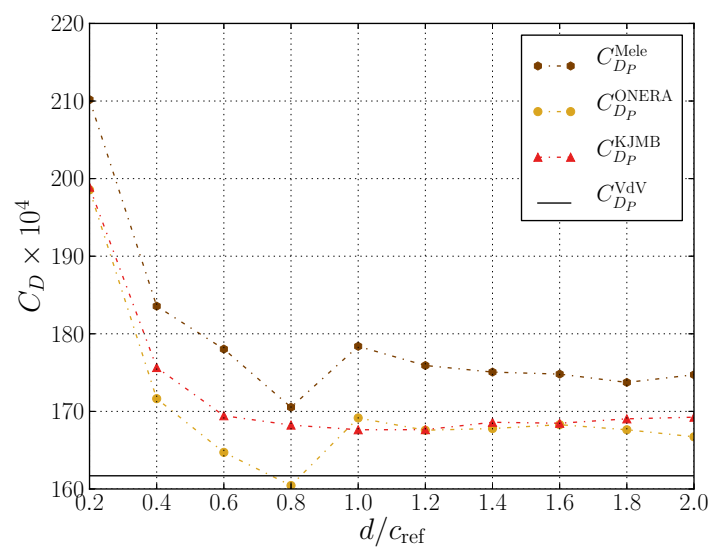

(b) Profile drag

Fig. 5 Lift-induced and profile drag predictions on the NASA CRM, $M_{\infty}=0.85, R e=5 \times 10^{6}, C_{L} \approx 0.5$ 
contribution again in better agreement with the prediction of Destarac and Van der Vooren's formulation. In particular, $C_{D_{P}}^{\mathrm{VdV}}$ is 17 drag counts lower than $C_{D_{P}}^{\mathrm{Mele}}$ and only 8 drag counts lower than $C_{D_{P}}^{\mathrm{ONERA}}$ for $d / c_{\text {ref }}=1$ (see Table 1 . The equivalence between the ONERA and KJMB formulations is however less visible for $d / c_{\text {ref }} \leq 1$. Actually, the bumpy aspect of the $C_{D_{P}}^{\mathrm{ONERA}}$ curve is attributable to numerical errors in the computation of the vorticity vector on the surface $S_{e}$. On the contrary, in the KJMB formulation, the bumpy character is not present in the curves representing $C_{D_{i}}^{\mathrm{KJMB}}$ and $C_{D_{P}}^{\mathrm{KJMB}}$ since there is no curl to compute in the integrals and therefore less inaccuracies. As a consequence, the physical evolutions of the lift-induced drag and the profile drag are better grasped. In fact, for $d / c_{\text {ref }} \geq 1.2$, the lift-induced drag starts decreasing in favor of the profile drag. This is the direct consequence of the physical diffusion and the numerical dissipation of the trailing vortices.

\begin{tabular}{lcccc}
\hline \hline Formulation & $C_{D_{i}} \times 10^{4}$ & $C_{D_{P}} \times 10^{4}$ & $C_{D} \times 10^{4}$ & $C_{L}$ \\
\hline Destarac and Van der Vooren [28] & 92.81 & 161.70 & 254.51 & - \\
Mele et al. [47] & 77.01 & 178.39 & 255.40 & 0.49852 \\
ONERA formulation & 86.29 & 169.17 & 255.46 & 0.49198 \\
KJMB formulation & 87.26 & 167.63 & 254.89 & 0.49698 \\
Near-field & - & - & 254.70 & 0.49946 \\
\hline \hline
\end{tabular}

Table 1 Comparison between the various force breakdown formulations on the NASA CRM, $M_{\infty}=0.85$, $R e=5 \times 10^{6}, C_{L} \approx 0.5$ and $d / c_{\text {ref }}=1$

In summary, it was shown that the ONERA formulation is indeed equivalent to the KJMB formulation, in mathematical and numerical terms. Those new formulations can be used in the aerodynamic performance evaluation of the NASA CRM wing-body configuration under cruise flight conditions. They also provide a better decomposition of the drag than that proposed by Mele et al. [47] since the lift-induced drag and profile drag predictions are in better adequation with the tried-and-tested Destarac and Van der Vooren's formulation [28]. In particular, the KJMB formulation is very interesting because it is an exact force breakdown which generalizes for the first time the classical far-field analyses in transonic viscous flows and can be used in civil aircraft applications.

The next subsection will investigate in more details the different contributors of $\boldsymbol{F}_{\text {BETZ. In particular, special }}$ attention will be devoted to the integral involving density variations in Eq. 347.

\section{Phenomenological analysis of $F_{\mathrm{BETZ}}$}

In the ONERA and KJMB formulations, it has been shown that the profile drag integral is equivalent to a compressible counterpart of Betz's formula. This formula has never been encountered before. It is then an opportunity to analyze how Betz's profile drag formula is modified in compressible flows. To do so, $\boldsymbol{F}_{\text {BETZ }}$ defined in Eq. (34) is now decomposed into

$$
\boldsymbol{F}_{\mathrm{BETZ}}=\oint_{S_{e}} \boldsymbol{\tau} \cdot \boldsymbol{n} \mathrm{d} S+\boldsymbol{F}_{\Delta p_{t}}+\boldsymbol{F}_{\Delta \rho}
$$

with

$$
\begin{aligned}
\boldsymbol{F}_{\Delta p_{t}} & =-\oint_{S_{e}} \Delta p_{t} \boldsymbol{n} \mathrm{d} S=\oint_{S_{e}}\left(p_{t \infty}-p_{t}\right) \boldsymbol{n} \mathrm{d} S \\
\boldsymbol{F}_{\Delta \rho} & =\frac{U_{\infty}^{2}}{2} \oint_{S_{e}} \Delta \rho \boldsymbol{n} \mathrm{d} S=\frac{U_{\infty}^{2}}{2} \oint_{S_{e}}\left(\rho-\rho_{\infty}\right) \boldsymbol{n} \mathrm{d} S
\end{aligned}
$$

where $\Delta p_{t}=p_{t}-p_{t \infty}$ and $\Delta \rho=\rho-\rho_{\infty}$. As mentioned earlier, Betz initially defined the profile drag as the total pressure loss in incompressible flows [13]. In compressible flows however, the contribution of $\boldsymbol{F}_{\Delta \rho}$ must be accounted for and his definition is not valid anymore. The aim is then to understand the physical role of $\boldsymbol{F}_{\Delta \rho}$ in compressible flows. For this purpose, it is necessary to find the link with the incompressible Betz's formula, denoted $\boldsymbol{F}_{\text {BETZ }}^{\text {incomp }}$

$$
\boldsymbol{F}_{\mathrm{BETZ}}=\boldsymbol{F}_{\mathrm{BETZ}}^{\mathrm{incomp}}+\oint_{S_{e}} \boldsymbol{\tau} \cdot \boldsymbol{n} \mathrm{d} S-\oint_{S_{e}}\left(\rho-\rho_{\infty}\right) \frac{q^{2}-U_{\infty}^{2}}{2} \boldsymbol{n} \mathrm{d} S
$$


with

$$
\boldsymbol{F}_{\mathrm{BETZ}}^{\mathrm{incomp}}=\oint_{S_{e}}\left[p_{t \infty}-\left(p+\frac{1}{2} \rho_{\infty} q^{2}\right)\right] \boldsymbol{n} \mathrm{d} S
$$

Hence, by adding up $\boldsymbol{F}_{\Delta p_{t}}$ and $\boldsymbol{F}_{\Delta \rho}$, the initial profile drag definition $\boldsymbol{F}_{\mathrm{BETZ}}^{\text {incomp }}$ developed by Betz [13] is recovered, plus a term involving the viscous stresses and the density and kinetic energy perturbations. This term is actually expected to vanish in the far field. In the following, the behavior of $\boldsymbol{F}_{\Delta p_{t}}, \boldsymbol{F}_{\Delta \rho}$ and $\boldsymbol{F}_{\text {BETZ }}^{\text {incomp }}$ with respect to the location of $S_{e}$ are examined.

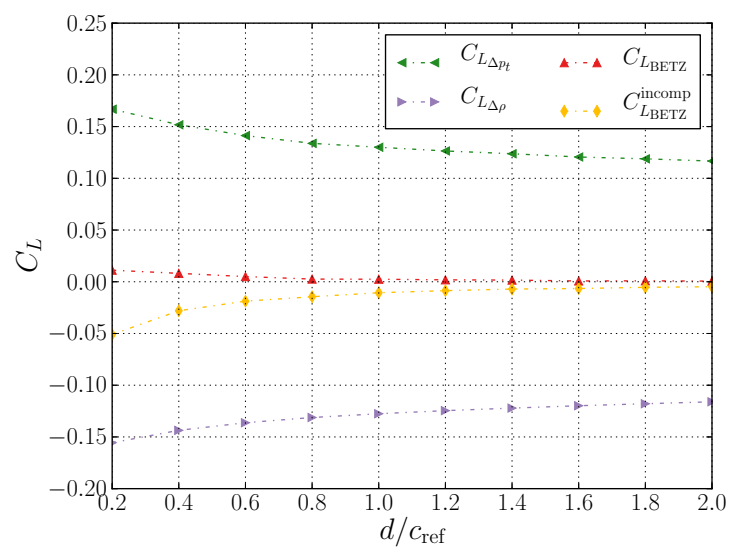

(a) Lift

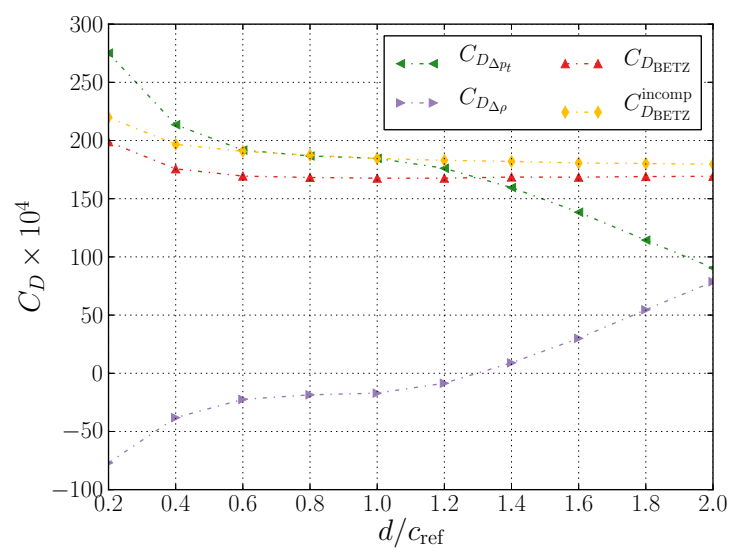

(b) Drag

Fig. 6 Lift and drag predictions of $\boldsymbol{F}_{\mathrm{BETZ}}$ on the NASA CRM, $M_{\infty}=0.85, R e=5 \times 10^{6}, C_{L} \approx 0.5$

The evolution of $\boldsymbol{F}_{\text {BETZ }}$ with respect to the size of the integration domain is represented in Fig 6 First of all, $\boldsymbol{F}_{\Delta p_{t}}$ yields a nonzero lift contribution, suggesting that total pressure variations are not involved solely in the drag in compressible flows. Besides, it is clear that $C_{L_{\Delta \rho} \rho}$ balances $C_{L_{\Delta p_{t}}}$. Hence, it indicates that the lift generated by total pressure variations comes from density variations. It is clearly seen in Fig 7 , the total pressure losses represented on the suction side of the wing (see Fig.7a in purple) exactly coincide with the decrease in density caused by the expansion in the same region (see Fig $7 \mathrm{~b}$ in blue). In fact, this expansion is responsible for a part of the lift in compressible flows. Moreover, $C_{L_{\mathrm{BETZ}}}^{\text {incomp }}$ quickly converges to zero and the $C_{L_{\mathrm{BETZ}}}$ curve as $d / c_{\text {ref }}$ is increased. It confirms that the lift contribution of the additional term on the right-hand side of Eq.(50) effectively disappears in the far field. In conclusion, in compressible flows, $C_{L_{\Delta \rho}}$ represents the lift contribution generated by the dynamic pressure variations of a fluid flowing at $U_{\infty}$.

Regarding the evolution of the drag, once again, $C_{D_{\Delta p t}}$ and $C_{D_{\Delta \rho}}$ display complementary evolutions, and for $d / c_{\text {ref }} \geq 0.6 C_{D_{\mathrm{BETZ}}}$ becomes constant. Indeed, the decrease in $C_{D_{\Delta p_{t}}}$ is always compensated by an increase in $C_{D_{\Delta \rho}}$. However, it is not obvious to explain this increase. In fact, $\frac{U_{\infty}^{2}}{2} \Delta \rho$ is integrated on the surface $S_{e}$, and $S_{e}$ is defined by its distance to $S_{b}$. Therefore, $S_{e}$ is a closed surface which boundaries always follow the shape of the aircraft. It is difficult to interpret the evolution of $C_{D_{\Delta \rho}}$ because $S_{e}$ intersects alternatively compression and expansion regions away from the wing leading and trailing edges (see Fig 8 .

In order to explain the evolution of $\boldsymbol{F}_{\Delta \rho}$, an analysis of $\frac{U_{\infty}^{2}}{2} \Delta \rho$ is necessary. It is seen in Fig $7 \mathrm{~b}$ that there is an increase in fluid density due to the compression at the nose of the aircraft, the wing leading edge and the bottom part of the aircraft. It generates a total pressure rise (see Fig $7 \mathrm{a}$ in orange) responsible for a drag contribution: it explains why $C_{D_{\Delta p_{t}}} \geq C_{D_{\mathrm{BETZ}}}$ and $C_{D_{\Delta \rho}} \leq 0$ for $d / c_{\text {ref }} \leq 1.3$. In the definition of $\boldsymbol{F}_{\mathrm{BETZ}}$, this contribution of $\boldsymbol{F}_{\Delta p_{t}}$ to the drag is however compensated by the negative contribution of $\boldsymbol{F}_{\Delta \rho}$, therefore suggesting that the density variations caused by the compression do not contribute to the profile drag. Outside the boundary layer, the fluid is then expanded (see Fig 88) and $\rho \leq \rho_{\infty}$. This expansion occurs below and above the aircraft cabin, suggesting that the contribution to the lift remains limited. The acceleration occuring downstream on both sides of the wing expands once again the fluid and is, as shown earlier, responsible for part of the lift in compressible flows. Later, the fluid is subject to another compression in the near 
wake of the wing. Then, the expansion occuring downstream is caused by the tapering of the fuselage at the tail before the fluid eventually undergoes a final compression. Inside the boundary layer and the wake, the density is always lower than $\rho_{\infty}$ because of the entropy generated by irreversible processes.
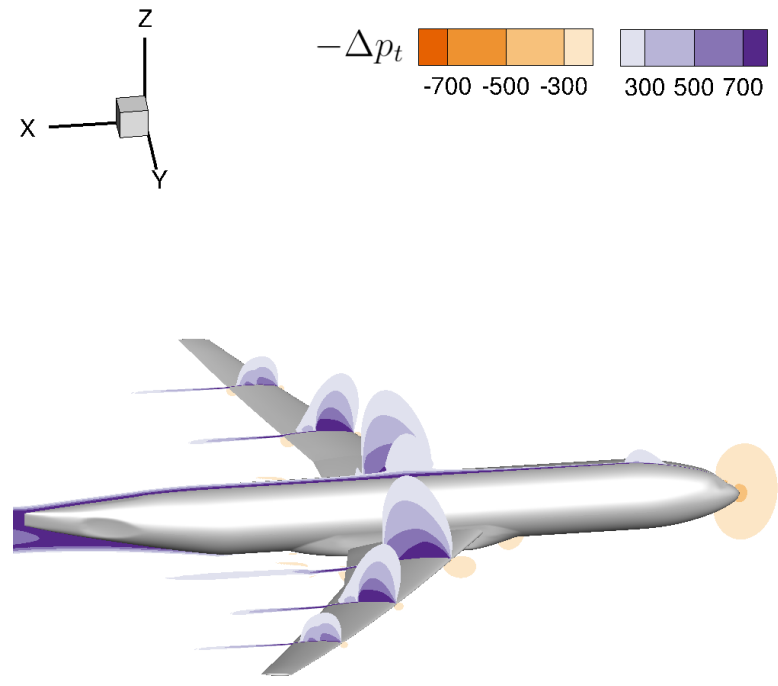

(a) $\Delta p_{t}$
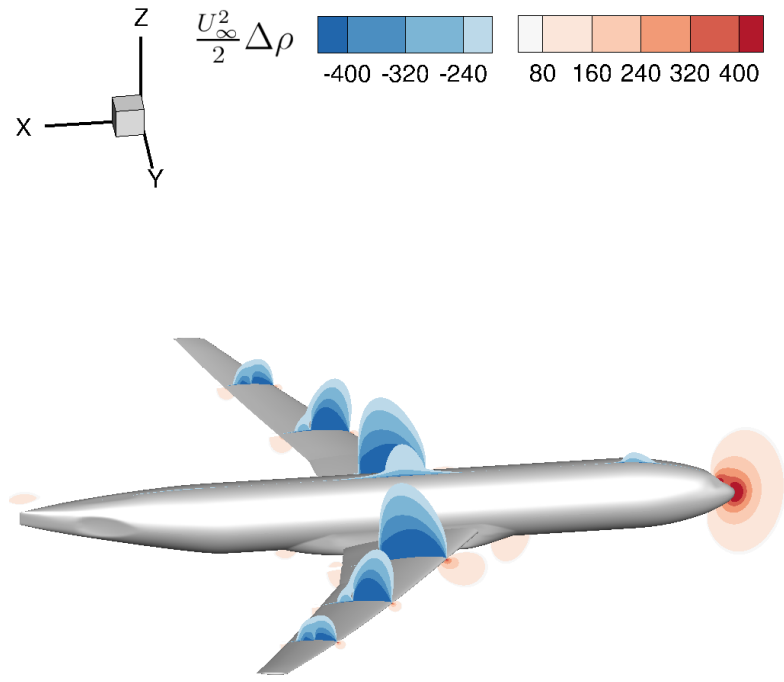

(b) $\frac{U_{\infty}^{2}}{2} \Delta \rho$

Fig. 7 Visualisations of the contributors of $\boldsymbol{F}_{\mathrm{BETZ}}$ on the NASA CRM, $M_{\infty}=0.85, R e=5 \times 10^{6}, C_{L} \approx 0.5$

The compressions and expansions identified in the preceding analysis are directly responsible for the variations of $\boldsymbol{F}_{\Delta \rho}$ with respect to $d / c_{\text {ref }}$ in Fig 6. Among else, it seems that the positive contribution to the $\operatorname{drag}$ seen for $d / c_{\text {ref }} \geq 1.3$ occurs when $S_{e}$ crosses in the same time the expansion region caused by the fuselage upstream of the wing (where $n_{x} \leq 0$ ) and the compression region near the tail of the fuselage (where $n_{x} \geq 0$ ). Anyways, the contribution $C_{D_{\Delta \rho}}$ accounts for total pressure variations induced by density variations and clearly balances $C_{D_{\Delta p_{t}}}$ : in the end, the profile drag corresponds to the total pressure losses induced only by losses in static pressure and kinetic energy in boundary

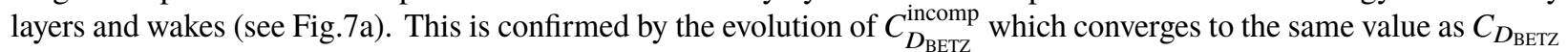
when $S_{e}$ extends further away. It also shows that the drag contribution of the additional term on the right-hand side of Eq. (50) disappears in the far field. In the end, the original Betz's formula $\boldsymbol{F}_{\text {BETZ }}^{\text {incomp }}$ [13] is still valid in compressible transonic flows, provided that the surface used for integration is located sufficiently far from the aircraft. 

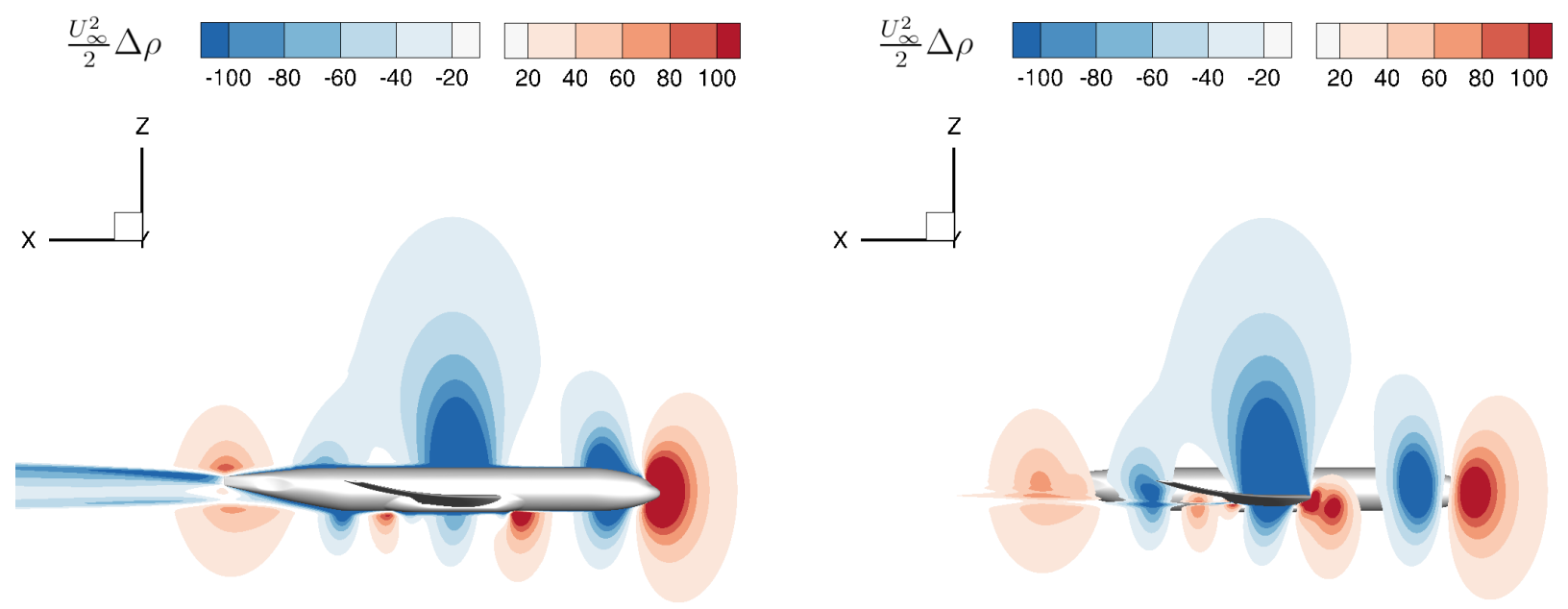

(a) $y / c_{\text {ref }}=0$

(b) $y / c_{\text {ref }}=0.5$
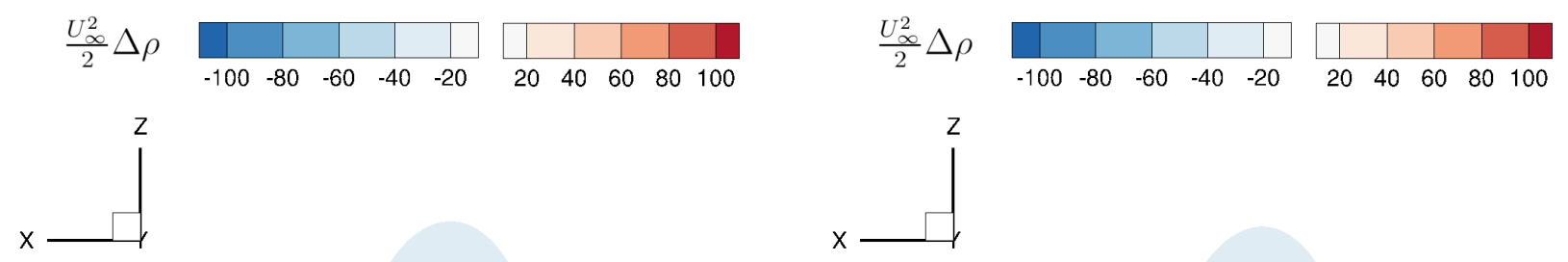

(c) $y / c_{\text {ref }}=1$

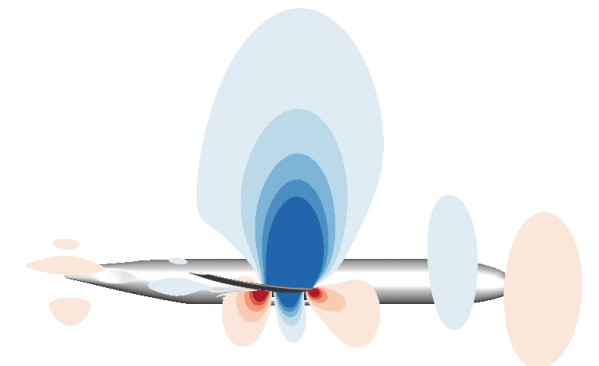

Fig. 8 Visualisations of $\frac{U_{\infty}^{2}}{2} \Delta \rho$ on the NASA CRM, $M_{\infty}=0.85, R e=5 \times 10^{6}, C_{L} \approx 0.5$ 


\section{Conclusion}

In the present study, a new formulation of the vortex-force theory was developed. This formulation now allows for a better understanding of the physics of lift and drag in compressible transonic flows. Indeed, this new formulation is equivalent to the classical far-field analyses developed by the pioneering aerodynamicists Kutta, Joukowski, Maskell and Betz. To be precise, it was shown that the new vortex-force theory encompasses these classical analyses in a single expression, and is able to provide an exact prediction and decomposition of the aerodynamic force. In compressible flows, the lift is still related to the circulation, and the lift-induced drag to the presence of trailing vortices. Moreover, it was proved that the profile drag represents the total pressure losses coming from losses in static pressure and kinetic energy only. Indeed, a nonvanishing contribution coming from density variations was emphasized in the total pressure losses. It comes from the expansions and compressions in the vicinity of the aircraft and contributes to part of the lift in compressible flows. Additionally, the new decomposition is completely independent of the reference point chosen for the computation of moments, and its application can be performed in regions closer to the aircraft where the grid is better refined.

A Reynolds-averaged-Navier-Stokes simulation on the NASA Common Research Model in transonic cruise flight conditions showed the maturity of the new formulation and its ability to predict and decompose the lift and the drag on an industrial aircraft configuration. Nevertheless, the decomposition of the profile drag into viscous and wave contributions remains an open issue, although it has already been attempted in the past, and further investigations are necessary in order to develop a robust definition for the wave drag.

\section{Appendix}

\section{A. Integral theorems}

Let $f$ be a differentiable vector field, $\Phi$ be a differentiable scalar field, $\Omega$ be a domain bounded by $\partial \Omega$ and $S$ be a surface bounded by $\partial S$. The derivative moment transformations (DMT) state that in an $\mathcal{N}$-dimensional space with $\mathcal{N}=2,3$ :

$$
\begin{aligned}
\int_{\Omega} \boldsymbol{f} \mathrm{d} v & =\frac{1}{\mathcal{N}-1} \int_{\Omega} \boldsymbol{r} \times(\nabla \times \boldsymbol{f}) \mathrm{d} v-\frac{1}{\mathcal{N}-1} \oint_{\partial \Omega} \boldsymbol{r} \times(\boldsymbol{n} \times \boldsymbol{f}) \mathrm{d} S \\
\int_{S} \Phi \boldsymbol{n} \mathrm{d} S & =-\frac{1}{\mathcal{N}-1} \int_{S} \boldsymbol{r} \times(\boldsymbol{n} \times \nabla \Phi) \mathrm{d} S+\frac{1}{\mathcal{N}-1} \oint_{\partial S} \Phi \boldsymbol{r} \times \mathrm{d} \boldsymbol{r}
\end{aligned}
$$

In the presence of a discontinuity, $f$ and $\Phi$ are no longer differentiable in the whole domain $\Omega$ or on the whole surface $S$. In this case, $\Omega$ is split into $\Omega_{1} \cup \Omega_{2}$ with $\Omega_{1}$ and $\Omega_{2}$ separated by a surface of discontinuity $S_{\text {dis }}$ and $S$ is split into $S_{1} \cup S_{2}$ with $S_{1}$ and $S_{2}$ separated by a line of discontinuity $L_{\text {dis }}$. Denoting $N$ the unit normal on the discontinuity pointing from region 1 to 2 , the derivative moment transformations now consist of

$$
\begin{aligned}
\int_{\Omega} \boldsymbol{f} \mathrm{d} v & =\frac{1}{\mathcal{N}-1} \int_{\Omega} \boldsymbol{r} \times(\nabla \times \boldsymbol{f}) \mathrm{d} v-\frac{1}{\mathcal{N}-1} \oint_{\partial \Omega} \boldsymbol{r} \times(\boldsymbol{n} \times \boldsymbol{f}) \mathrm{d} S+\frac{1}{\mathcal{N}-1} \int_{S_{\mathrm{dis}}} \boldsymbol{r} \times(\boldsymbol{N} \times \llbracket \boldsymbol{f} \rrbracket) \mathrm{d} S \\
\int_{S} \Phi \boldsymbol{n} \mathrm{d} S & =-\frac{1}{\mathcal{N}-1} \int_{S} \boldsymbol{r} \times(\boldsymbol{n} \times \nabla \Phi) \mathrm{d} S+\frac{1}{\mathcal{N}-1} \oint_{\partial S} \Phi \boldsymbol{r} \times \mathrm{d} \boldsymbol{r}-\frac{1}{\mathcal{N}-1} \int_{L_{\mathrm{dis}}} \llbracket \Phi \rrbracket \boldsymbol{r} \times \mathrm{d} \boldsymbol{r}
\end{aligned}
$$

Those identities are independent of $\boldsymbol{r}_{0}$ : a shift $\boldsymbol{r} \rightarrow \boldsymbol{r}+\boldsymbol{r}_{0}$ gives birth to several additional terms that cancel each other according to Gauss theorem. However, the right-hand side integrals individually depend on the choice of $\boldsymbol{r}_{0}$.

Similarly, it is possible to generalize the Gauss and Stokes theorems in the presence of discontinuities inside the integration domain. Let $\mathcal{F}$ be a tensor of any rank. Denoting $\llbracket \mathcal{F} \rrbracket=\mathcal{F}_{2}-\mathcal{F}_{1}$ and letting $\circ$ be any permissible operation:

$$
\begin{aligned}
\int_{\Omega} \nabla \circ \mathcal{F} \mathrm{d} v & =\oint_{\partial \Omega} \boldsymbol{n} \circ \mathcal{F} \mathrm{d} S-\int_{S_{\mathrm{dis}}} \boldsymbol{N} \circ \llbracket \mathcal{F} \rrbracket \mathrm{d} S \\
\int_{S}(\boldsymbol{n} \times \nabla) \circ \mathcal{F} \mathrm{d} S & =\oint_{\partial S} \mathrm{~d} \boldsymbol{r} \circ \mathcal{F}-\int_{L_{\mathrm{dis}}} \mathrm{d} \boldsymbol{r} \circ \llbracket \mathcal{F} \rrbracket
\end{aligned}
$$




\section{B. Equivalent expression of $F_{\mathrm{SW}}$}

By using the first generalized DMT Eq. A.3] and the generalized Gauss theorem Eq. A.5. in the shock wave region $\Omega_{\mathrm{sw}}$, it is possible to express $\boldsymbol{F}_{\mathrm{SW}}$ as follows:

$$
\begin{aligned}
\boldsymbol{F}_{\mathrm{SW}}= & \int_{\Omega_{\mathrm{sw}}} \nabla \cdot \boldsymbol{\tau} \mathrm{d} v-\oint_{\partial \Omega_{\mathrm{sw}}} \boldsymbol{\tau} \cdot \boldsymbol{n}_{\mathrm{sw}} \mathrm{d} S-\int_{\Omega_{\mathrm{sw}}}\left(\nabla p+\rho \nabla\left(\frac{q^{2}}{2}\right)\right) \mathrm{d} v \\
& +\frac{1}{\mathcal{N}-1} \int_{\Omega_{\mathrm{sw}}} \boldsymbol{r} \times\left(\nabla \rho \times \nabla\left(\frac{q^{2}}{2}\right)\right) \mathrm{d} v-\frac{1}{\mathcal{N}-1} \oint_{\partial \Omega_{\mathrm{sw}}} \boldsymbol{r} \times\left[\boldsymbol{n}_{\mathrm{sw}} \times\left(\nabla p+\rho \nabla\left(\frac{q^{2}}{2}\right)\right)\right] \mathrm{d} S
\end{aligned}
$$

It is important to remind here that $\boldsymbol{n}_{\mathrm{sw}}$ is the unit normal pointing outside $\Omega_{\mathrm{sw}}$. Using the right-hand side of the Navier-Stokes equations in Eq.99, $\nabla p+\rho \nabla\left(\frac{q^{2}}{2}\right)$ is replaced by $\nabla \cdot \tau-\rho \boldsymbol{l}$ in the volume integral of the first line of Eq.(B.1):

$$
\begin{aligned}
\boldsymbol{F}_{\mathrm{SW}}= & -\oint_{\partial \Omega_{\mathrm{sw}}} \boldsymbol{\tau} \cdot \boldsymbol{n}_{\mathrm{sw}} \mathrm{d} S+\int_{\Omega_{\mathrm{sw}}} \rho \boldsymbol{l} \mathrm{d} v+\int_{\Omega_{\mathrm{sw}}} \boldsymbol{m}_{\rho} \mathrm{d} v \\
& -\frac{1}{\mathcal{N}-1} \oint_{\partial \Omega_{\mathrm{sw}}} \boldsymbol{r} \times\left[\boldsymbol{n}_{\mathrm{sw}} \times\left(\nabla p+\rho \nabla\left(\frac{q^{2}}{2}\right)\right)\right] \mathrm{d} S
\end{aligned}
$$

where $\boldsymbol{m}_{\rho}$ is defined in Eq. (6). Let us have a closer look at the second line of Eq. B.2. $\partial \Omega_{\mathrm{sw}}$ is a closed surface which comprises the shock and its surroundings, such that it does not cross the shock discontinuity. Hence, no line of discontinuity crosses $\partial \Omega_{\mathrm{sw}}$. Having that

$$
\nabla p+\rho \nabla\left(\frac{q^{2}}{2}\right)=\nabla\left(p+\rho \frac{q^{2}}{2}\right)-\frac{q^{2}}{2} \nabla \rho
$$

and using the second DMT Eq. A.2,, $\boldsymbol{F}_{\mathrm{SW}}$ is now written in the following form:

$$
\begin{aligned}
\boldsymbol{F}_{\mathrm{SW}}= & \oint_{\partial \Omega_{\mathrm{sw}}}(p \boldsymbol{I}-\boldsymbol{\tau}) \cdot \boldsymbol{n}_{\mathrm{sW}} \mathrm{d} S+\int_{\Omega_{\mathrm{sw}}} \rho \boldsymbol{l} \mathrm{d} v+\int_{\Omega_{\mathrm{sw}}} \boldsymbol{m}_{\rho} \mathrm{d} v \\
& +\oint_{\partial \Omega_{\mathrm{sw}}} \rho \frac{q^{2}}{2} \boldsymbol{n}_{\mathrm{sw}} \mathrm{d} S+\frac{1}{\mathcal{N}-1} \oint_{\partial \Omega_{\mathrm{sw}}} \boldsymbol{r} \times\left(\boldsymbol{n}_{\mathrm{sw}} \times \frac{q^{2}}{2} \nabla \rho\right) \mathrm{d} S
\end{aligned}
$$

Let us now analyze the surface integral in the first line of Eq.B.3. The generalized Gauss theorem Eq. A.5. yields

$$
\oint_{\partial \Omega_{\mathrm{sw}}}(p \boldsymbol{I}-\boldsymbol{\tau}) \cdot \boldsymbol{n}_{\mathrm{sw}} \mathrm{d} S=\int_{\Omega_{\mathrm{sw}}}(\nabla p-\nabla \cdot \tau) \mathrm{d} v+\int_{\mathrm{SW}} \llbracket p \boldsymbol{I}-\tau \rrbracket \cdot \boldsymbol{N} \mathrm{d} S
$$

With the steady Navier-Stokes equations Eq.(9), $\nabla p-\nabla \cdot \tau$ is replaced by $-\nabla \cdot(\rho \boldsymbol{q} \boldsymbol{q})$ in the volume integral of Eq.(B.4). Using once again the generalized Gauss theorem Eq. A.5] in order to transform it into a surface integral, it is possible to write

$$
\oint_{\partial \Omega_{\mathrm{sw}}}(p \boldsymbol{I}-\boldsymbol{\tau}) \cdot \boldsymbol{n}_{\mathrm{sw}} \mathrm{d} S=-\oint_{\partial \Omega_{\mathrm{sw}}} \rho \boldsymbol{q}\left(\boldsymbol{q} \cdot \boldsymbol{n}_{\mathrm{sw}}\right) \mathrm{d} S+\underbrace{\int_{\mathrm{SW}} \llbracket \rho \boldsymbol{q} \boldsymbol{q}+p \boldsymbol{I}-\boldsymbol{\tau} \rrbracket \cdot \boldsymbol{N} \mathrm{d} S}_{=\mathbf{0}}
$$

The third term of Eq. B.5] is always zero since

$$
\llbracket \rho \boldsymbol{q} \boldsymbol{q}+p \boldsymbol{I}-\tau \rrbracket \cdot N=\mathbf{0}
$$

on the shock wave SW. The introduction of Eq. B.5) into Eq.(B.3) yields the desired form of $\boldsymbol{F}_{\mathrm{SW}}$ :

$$
\begin{aligned}
\boldsymbol{F}_{\mathrm{SW}}= & \int_{\Omega_{\mathrm{sw}}} \boldsymbol{m}_{\rho} \mathrm{d} v+\oint_{\partial \Omega_{\mathrm{sw}}}\left(\rho \frac{q^{2}}{2} \boldsymbol{n}_{\mathrm{sw}}-\rho \boldsymbol{q}\left(\boldsymbol{q} \cdot \boldsymbol{n}_{\mathrm{sw}}\right)\right) \mathrm{d} S \\
& +\frac{1}{\mathcal{N}-1} \oint_{\partial \Omega_{\mathrm{sw}}} \boldsymbol{r} \times\left(\boldsymbol{n}_{\mathrm{sw}} \times \frac{q^{2}}{2} \nabla \rho\right) \mathrm{d} S+\int_{\Omega_{\mathrm{sw}}} \rho \boldsymbol{l} \mathrm{d} v
\end{aligned}
$$




\section{Invariance of the ONERA formulation with respect to the reference point}

This appendix demonstrates that the new formulation of the vortex-force theory defined in Eqs. (41), (42) and (43) is naturally independent of the location of the reference point chosen for the computation of moment transformations. To do so, let us consider a shift $\hat{\boldsymbol{r}}=\boldsymbol{r}-\boldsymbol{r}_{0}$. In this case, it is possible to write the ONERA decomposition as follows:

$$
\begin{aligned}
\boldsymbol{F}_{\rho l}+\boldsymbol{F}_{m_{\rho}}^{\mathrm{trans}}-\boldsymbol{F}_{\nabla \rho}= & -\int_{\Omega \backslash \Omega_{\mathrm{sw}}} \rho \boldsymbol{l} \mathrm{d} v+\frac{1}{\mathcal{N}-1} \int_{\Omega \backslash \Omega_{\mathrm{sw}}} \hat{\boldsymbol{r}} \times\left(\nabla\left(\frac{q^{2}}{2}\right) \times \nabla \rho\right) \mathrm{d} v \\
& +\oint_{\partial \Omega_{\mathrm{sw}}}\left(\rho \frac{q^{2}}{2} \boldsymbol{n}_{\mathrm{sw}}-\rho \boldsymbol{q}\left(\boldsymbol{q} \cdot \boldsymbol{n}_{\mathrm{sw}}\right)\right) \mathrm{d} S+\frac{1}{\mathcal{N}-1} \oint_{\partial \Omega_{\mathrm{sw}}} \hat{\boldsymbol{r}} \times\left(\boldsymbol{n}_{\mathrm{sw}} \times \frac{q^{2}}{2} \nabla \rho\right) \mathrm{d} S \\
& -\frac{U_{\infty}^{2}}{2} \oint_{S_{e}}\left(\rho-\rho_{\infty}\right) \boldsymbol{n} \mathrm{d} S-\frac{1}{\mathcal{N}-1} \oint_{S_{e}} \hat{\boldsymbol{r}} \times\left(\boldsymbol{n} \times \frac{q^{2}}{2} \nabla \rho\right) \mathrm{d} S \\
& +\frac{\boldsymbol{r}_{0}}{\mathcal{N}-1} \times\left[\int_{\Omega \backslash \Omega_{\mathrm{sw}}} \nabla\left(\frac{q^{2}}{2}\right) \times \nabla \rho \mathrm{d} v-\oint_{S_{e}} \boldsymbol{n} \times \frac{q^{2}}{2} \nabla \rho \mathrm{d} S+\oint_{\partial \Omega_{\mathrm{sw}}} \boldsymbol{n}_{\mathrm{sw}} \times \frac{q^{2}}{2} \nabla \rho \mathrm{d} S\right]
\end{aligned}
$$

and

$$
\begin{aligned}
\boldsymbol{F}_{S_{e}}+\boldsymbol{F}_{\tau}+\boldsymbol{F}_{\nabla \rho}= & \oint_{S_{e}} \boldsymbol{\tau} \cdot \boldsymbol{n} \mathrm{d} S+\frac{1}{\mathcal{N}-1} \oint_{S_{e}} \hat{\boldsymbol{r}} \times(\boldsymbol{n} \times(\nabla \cdot \boldsymbol{\tau}-\rho \boldsymbol{l})) \mathrm{d} S \\
& +\frac{U_{\infty}^{2}}{2} \oint_{S_{e}}\left(\rho-\rho_{\infty}\right) \boldsymbol{n} \mathrm{d} S+\frac{1}{\mathcal{N}-1} \oint_{S_{e}} \hat{\boldsymbol{r}} \times\left(\boldsymbol{n} \times \frac{q^{2}}{2} \nabla \rho\right) \mathrm{d} S \\
& +\frac{\boldsymbol{r}_{0}}{\mathcal{N}-1} \times \oint_{S_{e}} \boldsymbol{n} \times\left(\nabla \cdot \boldsymbol{\tau}-\rho \boldsymbol{l}+\frac{q^{2}}{2} \nabla \rho\right) \mathrm{d} S
\end{aligned}
$$

Let us now analyze the last lines of the two preceding equations. In the last line of Eq.[C.1], it is possible to write the first integral as

$$
\int_{\Omega \backslash \Omega_{\mathrm{sw}}} \nabla\left(\frac{q^{2}}{2}\right) \times \nabla \rho \mathrm{d} v=\int_{\Omega \backslash \Omega_{\mathrm{sw}}} \nabla \times\left(\frac{q^{2}}{2} \nabla \rho\right) \mathrm{d} v
$$

and using the Gauss theorem for the cross product, it is possible to show that

$$
\int_{\Omega \backslash \Omega_{\mathrm{sw}}} \nabla \times\left(\frac{q^{2}}{2} \nabla \rho\right) \mathrm{d} v=\oint_{S_{e}} \boldsymbol{n} \times \frac{q^{2}}{2} \nabla \rho \mathrm{d} S-\oint_{\partial \Omega_{\mathrm{sw}}} \boldsymbol{n}_{\mathrm{sw}} \times \frac{q^{2}}{2} \nabla \rho \mathrm{d} S
$$

such that the last line of Eq. C.1] vanishes and

$$
\begin{aligned}
\boldsymbol{F}_{\rho l}+\boldsymbol{F}_{m_{\rho}}^{\mathrm{trans}}-\boldsymbol{F}_{\nabla \rho}= & -\int_{\Omega \backslash \Omega_{\mathrm{sw}}} \rho \boldsymbol{l} \mathrm{d} v+\frac{1}{\mathcal{N}-1} \int_{\Omega \backslash \Omega_{\mathrm{sw}}} \hat{\boldsymbol{r}} \times\left(\nabla\left(\frac{q^{2}}{2}\right) \times \nabla \rho\right) \mathrm{d} v \\
& +\oint_{\partial \Omega_{\mathrm{sw}}}\left(\rho \frac{q^{2}}{2} \boldsymbol{n}_{\mathrm{sw}}-\rho \boldsymbol{q}\left(\boldsymbol{q} \cdot \boldsymbol{n}_{\mathrm{sw}}\right)\right) \mathrm{d} S+\frac{1}{\mathcal{N}-1} \oint_{\partial \Omega_{\mathrm{sw}}} \hat{\boldsymbol{r}} \times\left(\boldsymbol{n}_{\mathrm{sw}} \times \frac{q^{2}}{2} \nabla \rho\right) \mathrm{d} S \\
& -\frac{U_{\infty}^{2}}{2} \oint_{S_{e}}\left(\rho-\rho_{\infty}\right) \boldsymbol{n} \mathrm{d} S-\frac{1}{\mathcal{N}-1} \oint_{S_{e}} \hat{\boldsymbol{r}} \times\left(\boldsymbol{n} \times \frac{q^{2}}{2} \nabla \rho\right) \mathrm{d} S
\end{aligned}
$$

To address the last line of Eq. C.2], one must first write the Navier-Stokes equations and notice that

$$
\oint_{S_{e}} \boldsymbol{n} \times\left(\nabla \cdot \boldsymbol{\tau}-\rho \boldsymbol{l}+\frac{q^{2}}{2} \nabla \rho\right) \mathrm{d} S=\oint_{S_{e}} \boldsymbol{n} \times \nabla p_{t} \mathrm{~d} S
$$

Using the Stokes theorem Eq.A.6 with $\partial S_{e}=\varnothing\left(S_{e}\right.$ is closed $)$ and knowing that $S_{e}$ is not crossed by any line of discontinuity, it is possible to show that

$$
\oint_{S_{e}} \boldsymbol{n} \times \nabla p_{t} \mathrm{~d} S=\mathbf{0}
$$

The last line of Eq. C.2. vanishes and

$$
\begin{aligned}
\boldsymbol{F}_{S_{e}}+\boldsymbol{F}_{\tau}+\boldsymbol{F}_{\nabla \rho}= & \oint_{S_{e}} \boldsymbol{\tau} \cdot \boldsymbol{n} \mathrm{d} S+\frac{1}{\mathcal{N}-1} \oint_{S_{e}} \hat{\boldsymbol{r}} \times(\boldsymbol{n} \times(\nabla \cdot \boldsymbol{\tau}-\rho \boldsymbol{l})) \mathrm{d} S \\
& +\frac{U_{\infty}^{2}}{2} \oint_{S_{e}}\left(\rho-\rho_{\infty}\right) \boldsymbol{n} \mathrm{d} S+\frac{1}{\mathcal{N}-1} \oint_{S_{e}} \hat{\boldsymbol{r}} \times\left(\boldsymbol{n} \times \frac{q^{2}}{2} \nabla \rho\right) \mathrm{d} S
\end{aligned}
$$


Therefore, the ONERA formulation naturally provides the same decomposition whatever the reference point $(\boldsymbol{r}$ or $\hat{\boldsymbol{r}})$ chosen for the computation of moment transformations.

\section{Grid convergence study}

This appendix presents a grid convergence study on the test case investigated in this paper. The effect of the grid sizing is sought on the total force computed by the ONERA formulation and a comparison is made with near-field results. The L4' grid of the NASA CRM [62] has been used since it is a well refined grid.

Four NASA CRM grids are tested in this convergence study: the L2' has a $y+=1.33$, the $\mathrm{L} 3^{\prime}$ has a $y+=1$, the $\mathrm{L} 4{ }^{\prime}$ has a $y+=0.67$ and the $\mathrm{L}^{\prime}$ has a $y+=0.5$.

\begin{tabular}{lcccc}
\hline \hline Grid level & $\mathrm{L}^{\prime}$ & $\mathrm{L}^{\prime}$ & $\mathrm{L}^{\prime}$ & $\mathrm{L}^{\prime}$ \\
\hline \multicolumn{5}{c}{$C_{D} \times 10^{4}$} \\
\hline$C_{D_{i}}^{\text {ONERA }}$ & 84.94 & 84.57 & 86.29 & 86.72 \\
$C_{D_{P}}^{\text {ONERA }}$ & 169.30 & 165.57 & 169.17 & 168.51 \\
$C_{D}^{\text {ONERA }}$ & 254.24 & 250.14 & 255.46 & 255.23 \\
$C_{D}^{\text {Near-field }}$ & 258.38 & 256.34 & 254.70 & 254.35 \\
\hline \multicolumn{5}{c}{$C_{L}$} \\
\hline$C_{L}^{\text {ONERA }}$ & 0.48583 & 0.48933 & 0.49198 & 0.49446 \\
$C_{L}^{\text {Near-field }}$ & 0.49967 & 0.49990 & 0.49946 & 0.49963 \\
\hline \hline
\end{tabular}

Table 2 Effect of the NASA CRM grid sizing on the ONERA force prediction and breakdown, $d / c_{\text {ref }}=1$

\section{Acknowledgments}

Part of this research was supported by a Direction Générale de l'Armement scholarship. The authors would also like to thank Ilias Petropoulos from ONERA, Meudon, France for providing the CFD solutions around the NASA CRM.

\section{References}

[1] Kutta, W. M., “Auftriebskräfte in Strömenden Flüssigkeiten," Illustrierte Aeronautische Mitteilungen, Vol. 6, No. 133, 1902, pp. $133-135$.

[2] Joukowski, N., “On annexed vortices,” Proc. Phys. Section of the Natural Science Society, Vol. 13, No. 2, 1906, pp. $12-25$.

[3] Bryant, L. W., and Williams, D. H., "V. An investigation of the flow of air an aerofoil of infinite span," Philosophical Transactions of the Royal Society of London. Series A, Containing Papers of a Mathematical or Physical Character, Vol. 225, No. 626-635, 1926, pp. 199-245. doi:10.1098/rsta.1926.0005.

[4] Prandtl, L., “Theory of lifting surfaces,” Tech. Rep. NACA TN10, National Advisory Committee for Aeronautics, 1919.

[5] Schmitz, S., "Finite Domain Viscous Correction to the Kutta-Joukowski Theorem in Incompressible Flow," AIAA Journal, Vol. 52, No. 9, 2014, pp. 2079-2083. doi:10.2514/1.J053114.

[6] Filon, L. N. G., "The forces on a cylinder in a stream of viscous fluid," Proceedings of the Royal Society of London. Series A, Containing Papers of a Mathematical and Physical Character, Vol. 113, No. 763, 1926, pp. 7-27. doi:10.1098/rspa.1926.0136.

[7] Drela, M., Flight vehicle aerodynamics, MIT press, 2014.

[8] Liu, L. Q., Zhu, J. Y., and Wu, J. Z., "Lift and drag in two-dimensional steady viscous and compressible flow," Journal of Fluid Mechanics, Vol. 784, 2015, pp. 304-341. doi:10.1017/jfm.2015.584.

[9] Liu, L. Q., Wu, J. Z., Su, W. D., and Kang, L. L., "Lift and drag in three-dimensional steady viscous and compressible flow," Physics of Fluids, Vol. 29, No. 11, 2017, p. 116105. doi:10.1063/1.4989747. 
[10] Kusunose, K., "Drag prediction based on a wake-integral method," 16th AIAA Applied Aerodynamics Conference, 1998, p. 2723. doi:10.2514/6.1998-2723.

[11] Kusunose, K., Crowder, J., and Watzlavick, R., "Wave drag extraction from profile drag based on a wake-integral method," 37th Aerospace Sciences Meeting and Exhibit, 1999, p. 275. doi:10.2514/6.1999-275.

[12] Méheut, M., and Bailly, D., "Drag-breakdown methods from wake measurements," AIAA journal, Vol. 46, No. 4, 2008 , pp. 847-862. doi:10.2514/1.29051.

[13] Betz, A., "A method for the direct determination of wing-section drag," Tech. Rep. NACA TM37, National Advisory Committee for Aeronautics, 1925.

[14] Taylor, G., "Note on the connection between the lift on an aërofoil in a wind and the circulation round it," Phil. Trans. R. Soc. London A, Vol. 225, 1926, pp. 238-246.

[15] Jones, B. M., The measurement of profile drag by the pitot-traverse method, HM Stationery Office, 1936.

[16] Maskell, E., "Progress Towards a Method for the Measurement of the Components of the Drag of a Wing of Finite Span," Tech. Rep. 72232, Procurement Executive, Ministry of Defence, Royal Aircraft Establishment, 1972.

[17] Cummings, R. M., Giles, M. B., and Shrinivas, G., "Analysis of the elements of drag in three-dimensional viscous and inviscid flows," 14th Applied Aerodynamics Conference, 1996, p. 2482. doi:10.2514/6.1996-2482.

[18] Giles, M. B., and Cummings, R. M., "Wake integration for three-dimensional flowfield computations: theoretical development," Journal of aircraft, Vol. 36, No. 2, 1999, pp. 357-365. doi:10.2514/2.2465.

[19] Hunt, D. L., Cummings, R. M., and Giles, M. B., "Wake integration for three-dimensional flowfield computations: Applications," Journal of aircraft, Vol. 36, No. 2, 1999, pp. 366-373. doi:10.2514/2.2466.

[20] Van Dam, C. P., "Recent experience with different methods of drag prediction," Progress in Aerospace Sciences, Vol. 35, No. 8, 1999, pp. 751-798. doi:10.1016/S0376-0421(99)00009-3.

[21] Kroo, I., "Drag due to lift: concepts for prediction and reduction," Annual review of fluid mechanics, Vol. 33, No. 1, 2001, pp. 587-617. doi:10.1146/annurev.fluid.33.1.587.

[22] Kusunose, K., and Crowder, J. P., "Extension of wake-survey analysis method to cover compressible flows," Journal of aircraft, Vol. 39, No. 6, 2002, pp. 954-963. doi:10.2514/2.3048.

[23] Chao, D., and Van Dam, C., "Wing drag prediction and decomposition," Journal of Aircraft, Vol. 43, No. 1, 2006 , pp. 82-90. doi:10.2514/1.12311.

[24] Ueno, M., Yamamoto, K., Tanaka, K., Murayama, M., and Tognaccini, R., "Far-field drag analysis of NASA Common Research Model simulation," Journal of Aircraft, Vol. 50, No. 2, 2013, pp. 388-397. doi:10.2514/1.C031766.

[25] Van Der Vooren, J., and Slooff, J., "CFD-based drag prediction: state-of-the-art, theory, prospects," National Aerospace Lab., NLR, TP 90247 U, 1992.

[26] Paparone, L., and Tognaccini, R., "Computational Fluid Dynamics-Based Drag Prediction and Decomposition," AIAA Journal, Vol. 41, No. 9, 2003, pp. 1647-1657. doi:10.2514/2.7300.

[27] Destarac, D., "Far-field/near-field drag balance and applications of drag extraction in CFD," VKI Lecture Series, Vol. 2, 2003, pp. 3-7.

[28] Destarac, D., and Van Der Vooren, J., "Drag/thrust analysis of jet-propelled transonic transport aircraft; definition of physical drag components," Aerospace science and technology, Vol. 8, No. 6, 2004, pp. 545-556. doi:10.1016/j.ast.2004.03.004.

[29] Fan, Y., and Li, W., "Review of far-field drag decomposition methods for aircraft design," Journal of Aircraft, Vol. 56, No. 1, 2019, pp. 11-21. doi:10.2514/1.C034781.

[30] Oswatitsch, K., Der Luftwiderstand als Integral des Entropiestromes, Vandenhoeck \& Ruprecht, 1945.

[31] Oswatitsch, K., Gas dynamics, Vol. 1, Academic Press, 1956.

[32] Spalart, P. R., "On the far wake and induced drag of aircraft," Journal of Fluid Mechanics, Vol. 603, 2008 , pp. 413-430. doi:10.1017/S0022112008001146. 
[33] Schmitz, S., and Coder, J. G., "Inviscid circulatory-pressure field derived from the incompressible Navier-Stokes equations," AIAA Journal, Vol. 53, No. 1, 2014, pp. 33-41. doi:10.2514/1.J053140.

[34] Schmitz, S., "Drag Decomposition Using Partial-Pressure Fields in the Compressible Navier-Stokes Equations," AIAA Journal, Vol. 57, No. 5, 2018, pp. 2030-2038. doi:10.2514/1.J057701.

[35] Coder, J. G., and Schmitz, S., "Thermodynamic Decomposition of Compressible Wave Drag in the Euler Equations," AIAA Aviation 2019 Forum, 2019, p. 2958.

[36] Wu, J. Z., Liu, L. Q., and Liu, T. S., "Fundamental theories of aerodynamic force in viscous and compressible complex flows," Progress in Aerospace Sciences, 2018. doi:10.1016/j.paerosci.2018.04.002.

[37] Saffman, P. G., Vortex dynamics, Cambridge university press, 1992.

[38] Wu, J. Z., Ma, H. Y., and Zhou, M. D., Vorticity and vortex dynamics, Springer Science \& Business Media, 2007.

[39] Wu, J. Z., Lu, X. Y., and Zhuang, L. X., "Integral force acting on a body due to local flow structures," Journal of Fluid Mechanics, Vol. 576, 2007, pp. 265-286. doi:10.1017/S0022112006004551.

[40] Liu, L. Q., Wu, J. Z., Shi, Y. P., and Zhu, J. Y., "A dynamic counterpart of Lamb vector in viscous compressible aerodynamics," Fluid Dynamics Research, Vol. 46, No. 6, 2014, p. 061417. doi:10.1088/0169-5983/46/6/061417.

[41] Liu, L. Q., Shi, Y. P., Zhu, J. Y., Su, W. D., Zou, S. F., and Wu, J. Z., "Longitudinal-transverse aerodynamic force in viscous compressible complex flow," Journal of Fluid Mechanics, Vol. 756, 2014, pp. 226-251. doi:10.1017/jfm.2014.403.

[42] Marongiu, C., and Tognaccini, R., "Far-field analysis of the aerodynamic force by Lamb vector integrals," AIAA Journal, Vol. 48, No. 11, 2010, pp. 2543-2555. doi:10.2514/1.J050326.

[43] Mele, B., and Tognaccini, R., "Aerodynamic force by Lamb vector integrals in compressible flow," Physics of Fluids, Vol. 26, No. 5, 2014, p. 056104. doi:10.1063/1.4875015.

[44] Marongiu, C., Tognaccini, R., and Ueno, M., "Lift and lift-induced drag computation by Lamb vector integration," AIAA Journal, Vol. 51, No. 6, 2013, pp. 1420-1430. doi:10.2514/1.J052104.

[45] Mele, B., Ostieri, M., and Tognaccini, R., "Vorticity based breakdown of the aerodynamic force in three-dimensional compressible flows," AIAA Journal, Vol. 54, No. 4, 2016, pp. 1198-1208. doi:10.2514/1.J054363.

[46] Yang, Y. T., Zhang, R. K., An, Y. R., and Wu, J. Z., "Steady vortex force theory and slender-wing flow diagnosis," Acta Mechanica Sinica, Vol. 23, No. 6, 2007, pp. 609-619. doi:10.1007/s10409-007-0107-0.

[47] Mele, B., Ostieri, M., and Tognaccini, R., "Aircraft lift and drag decomposition in transonic flows," Journal of Aircraft, Vol. 54, No. 5, 2017, pp. 1933-1944. doi:10.2514/1.C034288.

[48] Ostieri, M., and Tognaccini, R., “On a recently proposed vorticity-based definition of wave drag," Journal of Aircraft, Vol. 55, No. 6, 2018, pp. 2521-2523. doi:10.2514/1.C034820.

[49] Zou, S. F., Wu, J. Z., Gao, A. K., Liu, L. Q., Kang, L. L., and Shi, Y. P., "On the concept and theory of induced drag for viscous and incompressible steady flow," Physics of Fluids, Vol. 31, No. 6, 2019, p. 065106. doi:10.1063/1.5090165.

[50] Kang, L. L., Russo, L., Tognaccini, R., Wu, J. Z., and Su, W. D., "Aerodynamic Force Breakdown in Reversible and Irreversible Components by Vortex Force Theory,” AIAA Journal, Vol. 57, No. 11, 2019, pp. 4623-4638. doi:10.2514/1.J058379.

[51] Fournis, C., Bailly, D., and Tognaccini, R., "A reference point invariant Lamb vector based aerodynamic force breakdown in steady compressible flows," AIAA Scitech 2020 Forum, 2020. doi:10.2514/6.2020-1996.

[52] Fournis, C., Bailly, D., and Tognaccini, R., "Definition of an Invariant Lamb-Vector-Based Aerodynamic Force Breakdown Using Far-Field Flow Symmetries,” AIAA Journal, Vol. 59, No. 1, 2021, pp. 34-48. doi:10.2514/1.J059591.

[53] Wu, J. Z., Ma, H. Y., and Zhou, M. D., Vortical flows, Vol. 28, Springer, 2015. doi:10.1007/978-3-662-47061-9.

[54] Landau, L., and Lifshitz, E., Course of theoretical physics. vol. 6: Fluid mechanics, A. Wheaton \& Co., 1959.

[55] John D. Anderson, J., Fundamentals of aerodynamics, MacGraw-Hill, Inc., 1991.

[56] Chang, C.-C., and Lei, S.-Y., “An analysis of aerodynamic forces on a delta wing,” Journal of Fluid Mechanics, Vol. 316, 1996, pp. 173-196. doi:10.1017/S0022112096000493. 
[57] Chang, C.-C., Su, J.-Y., and Lei, S.-Y., "On aerodynamic forces for viscous compressible flow," Theoretical and computational fluid dynamics, Vol. 10, No. 1-4, 1998, pp. 71-90. doi:10.1007/s001620050051.

[58] Fournis, C., Bailly, D., and Tognaccini, R., "Compressibility Correction to Kutta-Joukowski and Maskell Formulas Using Vortex-Force Theory,” AIAA Journal, Vol. 59, No. 2, 2021, pp. 758-763. doi:10.2514/1.J059710.

[59] Kusunose, K., and Crowder, J., "Physical properties of Maskell's induced drag integral," 39th Aerospace Sciences Meeting and Exhibit, 2001, p. 421. doi:10.2514/6.2001-421.

[60] Schmitt, V., and Destarac, D., "Recent progress in drag prediction and reduction for civil transport aircraft at ONERA," 36th AIAA Aerospace Sciences Meeting and Exhibit, 1998, p. 137. doi:10.2514/6.1998-137.

[61] Cambier, L., Heib, S., and Plot, S., "The Onera elsA CFD software: input from research and feedback from industry,” Mechanics \& Industry, Vol. 14, No. 3, 2013, pp. 159-174. doi:10.1051/meca/2013056.

[62] Hue, D., "Fifth drag prediction workshop: ONERA investigations with experimental wing twist and laminarity," Journal of Aircraft, Vol. 51, No. 4, 2014, pp. 1311-1322. doi:10.2514/1.C032438.

[63] Vassberg, J., "A Unified Baseline Grid about the Common Research Model Wing/Body for the Fifth AIAA CFD Drag Prediction Workshop," 29th AIAA Applied Aerodynamics Conference, 2011, p. 3508. doi:10.2514/6.2011-3508. 